Modelling Simul. Mater. Sci. Eng., final accepted manuscript

\title{
Linking simulations and experiments for the multiscale tracking of thermally induced martensitic phase transformation in NiTi SMA
}

\author{
Sourav Gur $^{1}$ and George N. Frantziskonis ${ }^{1,2}$ \\ ${ }^{1}$ Civil Engineering and Engineering Mechanics, University of Arizona, Tucson, AZ \\ 85721 USA \\ ${ }^{2}$ Materials Science and Engineering, University of Arizona, Tucson, AZ 85721 USA \\ Email: frantzis@email.arizona.edu
}

\begin{abstract}
Martensitic phase transformation in NiTi shape memory alloys (SMA) occurs over a hierarchy of spatial scales, as evidenced from observed multiscale patterns of the martensitic phase fraction, which depend on the material microstructure and on the size of the SMA specimen. This paper presents a methodology for the multiscale tracking of the thermally induced martensitic phase transformation process in NiTi SMA. Fine scale stochastic phase field simulations are coupled to macroscale experimental measurements through the compound wavelet matrix method (CWM). A novel process for obtaining CWM fine scale wavelet coefficients is used that enhances the effectiveness of the method in transferring uncertainties from fine to coarse scales, and also ensures the preservation of spatial correlations in the phase fraction pattern. Size effects, well-documented in the literature, play an important role in designing the multiscale tracking methodology. Molecular dynamics (MD) simulations are employed to verify the phase field simulations in terms of different statistical measures and to demonstrate size effects at the nanometer scale. The effects of thermally induced martensite phase fraction uncertainties on the constitutive response of NiTi SMA is demonstrated.
\end{abstract}

Keywords: NiTi SMA, martensitic phase transformation, phase field simulations, multiscale coupling, predictive CWM, size effect

\section{Introduction}

The past few decades have witnessed an ever increasing interest in multiscale predictive modeling of science and engineering problems, fueled by the desire to understand problems across multiple physical scales. As a result, the framework of multiscale/ multiphysics (MSMP) methods has emerged [1-3] which, in the area of materials behavior includes two categories, namely, sequential and concurrent. In sequential multiscaling methods [1,2], simulations are performed at a fine/ atomic scale and the fundamental material properties obtained at these scales are used in the coarse scale/ continuum level simulations. In general, fine scale simulations are inherently stochastic, thereby an appropriate quantification of uncertainty associated with material properties requires a large number of simulations, and each simulation is typically by itself computationally demanding. In concurrent multiscaling methods [4-7], simulations are performed simultaneously in different spatial regions using different sub methods. More accurate fine scale simulations are performed in regions where such accuracy is needed, and are connected to regions where coarse scale simulations are performed. Alternatively, concurrent simulations can be performed at various scales, and then linked 
together through a scale-wise data fusion process, such as the compound wavelet matrix method (CWM) [8,9], which uses the inherent multiresolution capabilities of wavelet bases to epitomize a physical process in a multiscale fashion. A relevant review paper [10] provides citation of over 200 works on multiscale modeling.

Simulation of martensitic phase transformation in SMAs such as NiTi is computationally challenging due to the intricate stochasticity associated with the formation of different martensite phases (different martensite variants) occurring over a hierarchy of spatiotemporal scales $[11,12]$. The developed mechanisms in terms of internal strain or energy associated with the phase transformation from the parent austenite phase to the various martensite variants varies with the variants, thus, quantification of all different phase transformation mechanisms requires a large number of computationally burdensome fine scale simulations. Thus, bridging the different scales through sequential multiscaling requires a large number of computational simulations such that the scale dependent statistical behavior of phase transformation and the associated mechanisms are captured accurately. The CWM concurrent multiscale methodology is particularly appealing for tracking the thermally induced martensitic phase in NiTi SMA since it is capable of bridging information from different scales obtained from different scale specific simulations and/or experiments, finally yielding a compound multiscale representation of the process.

Wavelet based methods form a viable concurrent multiscale modeling toolbox [1-3,6-9]. They substantially reduce the computational overhead, without compromising accuracy, required to transfer information from one physical scale to another. A recent review paper [13] describes the application of wavelet based methods for solving reaction-diffusion (RD) problems in science and engineering fields and cites almost 140 relevant publications. Other than those general review papers, several field-specific reviews for chemical engineering [14], reactor technology [15] and surface science [16] are available. Bindal et al [17] employed a waveletbased method to formulate an adaptive multiscale solution strategy for dynamical systems in chemical processes. Stundzia and Lumsden [18] used a stochastic process for simulation of RD problems and Burger and Ruiz-Baier [19] performed multi-resolution simulation of RD systems with strong degeneracy. Research on the multiscaling approaches such as gap tooth and time stepping $[20,21]$ and wavelet based multiscaling, both in spatial scaling [5,8,22,23] and time scaling [7,9] are also reported. Muralidharan et al [6] introduced the dynamic compound wavelet matrix (dCWM) method as an adaptive technique in multiscale/ multiphysics (MSMP) problems, while Frantziskonis et al [7] proposed a time-parallel compound wavelet matrix (tpCWM) method as a time accelerating scheme for MSMP problems. In those studies, wavelets have been employed as a multiscale interface, rather than as a pure multiresolution toolbox since resolution translates to scale or scale of observation. Wavelet-based techniques offer the advantage that they preserve the non-stationary features in the fine scale data which can be lost or significantly distorted by other types of infinite basis transforms (e.g., Fourier analysis). All of these make the wavelet based CWM method a viable technique for coupling incongruent simulation methods (due to different levels of theory) that discourse the same process. Thus the CWM method has been used in various field of multiscale problems such as grain-growth and microstructure evolution, heterogeneous porosity and inclusions, dispersion problems and diffusion from a reactive boundary.

Here, the compound wavelet matrix (CWM) method is considered as a spatial multiscaling interface for coupling the martensite phase fraction evolution as obtained through a phase field 
fine/stochastic simulation framework and deterministic coarse scale experimental data. In particular, within a small spatial domain, fluctuating martensite phase fraction values are obtained through the rather computationally expensive phase field simulation method, which is inherently stochastic and thus involves uncertainties. Whereas, for large spatial domains information on martensite phase fraction is obtained from available experimental data. The fine (phase field) and coarse (deterministic) data are combined by employing a predictive compound wavelet matrix (CWM) method to yield the coupled multiscale behavior of martensitic phase transformation over the entire extent of the spatial domain. Through the CWM method, material uncertainties associated with the martensite phase fraction evolution at fine scales is propagated to coarse scales.

The multiscale framework inherently addresses size effects in the martensite phase fraction. For this reason, i.e. to observe the size effects over smaller scales, molecular dynamics (MD) simulations are performed. In general, MD can be actively invoked in the multiscale coupling process, however this is not pursued in the present study. Rather, MD simulations are employed to verify the phase field simulations in terms of different statistical measures (mean and standard deviation of martensite phase fraction) and most importantly to demonstrate the size effects at the nanometer scale. Finally, the impact of uncertainty on the stress-strain response of NiTi shape memory alloy is demonstrated.

The experimental data used herein are from polycrystalline NiTi SMAs, which contain interfacial energy and grain boundary energy in addition to the free energy in single crystal NiTi SMAs. As is known and also demonstrated in this work, there is a strong interplay between the average grain size in polycrystalline NiTi and the size of the specimen when it comes to thermally induced martensitic phase transformation. This interplay, which has received little attention, perhaps because of the difficulties in examining it, is challenging to address, so, in this study, first, to isolate the size effects, single crystal phase field (PhF) simulations are performed. Then the interplay is addressed in a later section recognizing that more innovative work is needed to address it robustly.

\section{Simulations and experimental data}

Three different methodologies operating at different scale ranges are employed, i.e. molecular dynamics (MD), phase field (PhF), and macroscopic experimental measurements. The main implementation of the CWM methodology employs the PhF and the macro scales, and the MD simulations are used to verify the PhF methodology and to address size effects in martensite phase fraction at atomistic scales. A brief description of the simulation methods is given since they are well-established and described in details in the literature. Simulations are performed in two spatial dimensions (2-D), yet the framework is extendable to 3-D. The implications of the 2-D simulations are discussed throughout the paper.

\subsection{Molecular dynamics (MD) simulations}

An effective MD framework for NiTi based on the Finnis-Sinclair many body interatomic potential has been proposed and examined in detail. Initially this potential was proposed by Lai and Liu [24], based on the embedded-atom method (EAM) considering a second-moment approximation of tight-binding theory (TBSMA). For the self-containment, a brief and relevant description of the MD simulation procedure and characterization of MD simulation cells is 
provided here, yet more detailed information can be found elsewhere [25-27]. According to this potential, the total energy of the system over all atoms is expressed as

$$
E^{(t)}=\sum_{i}\left(E_{i}^{(R)}+E_{i}^{(B)}\right)
$$

In (1), the first term in the right hand side indicates the pairwise interaction energy due to BornMayer repulsion, which stabilizes the crystal, and the second term represents many-body effects, considering second moment for the electron density. Recently the many-body interaction part of Lai and Liu potential has been modified by Zhong et al [25,28], where the terms in (1) are expressed as

$$
E_{i}^{(B)}=\left\{\begin{array}{c}
E_{i}^{(R)}=\sum_{i \neq j} A_{\alpha \beta} \exp \left[-p_{\alpha \beta}\left(\frac{r_{i j}}{d_{\alpha \beta}}-1\right)\right] \\
-\sqrt{\sum_{i \neq j} \xi_{\alpha \beta}^{2} \exp \left[-2 q_{\alpha \beta}\left(\frac{r_{i j}}{d_{\alpha \beta}}-1\right)\right]} \text { for } r_{i j} \leq r_{1} \\
-\sqrt{c_{0, \alpha \beta}+\sum_{i \neq j}\left[c_{1, \alpha \beta}\left(\frac{r_{i j}}{r_{1}}-1\right)+c_{2, \alpha \beta} r_{1}^{2}\left(\frac{r_{i j}}{r_{1}}-1\right)^{2}+c_{3, \alpha \beta} r_{1}^{3}\left(\frac{r_{i j}}{r_{1}}-1\right)^{3}\right]} \text { for } r_{1}<r_{i j} \leq r_{c}
\end{array}\right.
$$

In (2) and (3), $i$ and $j$ denote atoms, and $\alpha, \beta$ denote type of atoms ( $\mathrm{Ni}$ or Ti). A detailed description of the parameters involved is provided in Zhong et al [25].

Quasi-static MD simulations were performed in LAMMPS [29], for temperature driven transformation and for 2-D square films with free (non-periodic) boundary conditions in both directions. Seven different sizes were simulated, i.e. of side 2, 4, 8, 16, 32, 64, and $128 \mathrm{~nm}$, and the film thickness has been kept constant at $2 \mathrm{~nm}$ for all sizes. The temperature range was $50-750 \mathrm{~K}$, with an interval of $10 \mathrm{~K}$ within the temperature range of $250-550 \mathrm{~K}$ and with $50 \mathrm{~K}$ interval for the remaining temperature range. In the simulations, first, a pristine austenite B2 2-D, NiTi thin film sample is relaxed using a stress controlled conjugate gradient energy minimization method. Then the relaxed sample is given a velocity distribution corresponding to a particular temperature and held for $360 \mathrm{ps}$, under an NPT stabilization where the systems pressure is kept constant at 0 bars conditions. Then the NPT stabilized sample is subjected to a constant volume (NVT) conditions for an additional $240 \mathrm{ps}$. In each case of NPT or NVT stabilization, the adopted time step is $0.0005 \mathrm{ps}$. During simulation the temperature is controlled by a Nose-Hoover thermostat and Berendsen barostat. In order to remove the effects of thermal fluctuations the atomic coordinates were averaged over $20 \mathrm{ps}$. It was verified that the systems were well-equilibrated and the fluctuations in total energy and temperature were smaller than $0.001 \mathrm{eV} /$ atom and $0.50 \mathrm{~K}$, respectively.

To distinguish austenite B2 from martensite B19' and its variants, the bond length based order parameter proposed by Mutter and Nielaba $[26,30]$ was adopted. It is expressed as

$$
\chi\left(d_{0}, d_{1}\right)=\frac{d_{0}\left(d^{B 2}+d_{1}^{B 19^{\prime}}\right)-d_{1}\left(d^{B 2}+d_{0}^{B 19^{\prime}}\right)}{d^{B 2}\left(d_{0}^{B 19^{\prime}}-d_{1}^{B 19^{\prime}}\right)}
$$


Detailed description of the different parameters is provided in [30]. This order parameter has a value near +1.0 for the martensite B19' phase and -1.0 for the austenite B2 phase. To distinguish between the austenite B2 phases from martensite B19' phase, atoms with order parameter greater than +0.75 correspond to martensite B19' phase and less than -0.75 to austenite B2 phase. It has been observed that the bond length based order parameter for austenite B2 and martensite B19' phase exhibits a Gaussian distribution around +1.0 and -1.0 , respectively [30]. Such distribution for the order parameter arises primarily from the effects of thermal expansion or contraction on the bond length of the phases at different temperature. At every temperature and size, the martensite phase fraction was calculated by taking the ratio of intensity and spread in the Gaussian distributions. Further details are provided in the supplementary material.

\subsection{Phase field $(\mathrm{PhF})$ simulation method}

Phase field $(\mathrm{PhF})$ simulation of different types of first-order solid-solid phase transformations is a widely used and validated simulation methodology [31-42]. PhF is employed here using the Ginzburg-Landau (GL) type polynomial energy function. In particular, the recently proposed model by Zhong and Zhu [31] is adopted to simulate the evolution of martensite phase fraction at meso scales. A brief description of the $\mathrm{PhF}$ simulation process is given in section 2.2.1.

The same temperature ranges and intervals as those in the MD simulations are used. Seven different sizes of 2-D, NiTi thin films are simulated, i.e. of side 32, 64, 128, 256, 512, 1024

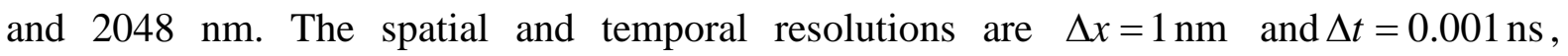
respectively. Each simulation is performed for a total time of $20 \mathrm{~ns}$ and, out of that, for the first $5 \mathrm{~ns}$, the simulation is perturbed by uncorrelated Langevin noise. Convergence of the simulation process is ensured by iteration (flow chart provided in the supplementary material) by comparing different statistical measures (mean, standard deviation and higher order moments (skewness and kurtosis)) of the martensite phase fraction between two consecutive time steps. The difference in value between two consecutive time steps i.e. $\Delta_{X}=\operatorname{abs}\left(X_{n+1}-X_{n}\right) / X_{n}$, where $X$ stands for mean or standard deviation or skewness or kurtosis of the martensite phase fraction, is compared to a tolerance limit chosen as $\left(\delta_{X}=0.001\right)$. Iterations stop once $\Delta_{X} \leq \delta_{X}$ is achieved.

\subsubsection{The phase field model}

Below the martensite start temperature $\mathrm{M}_{\mathrm{s}}$, the parent austenite B2 phase transforms into twelve different variants of martensite B19' phase [31]. In PF simulations, the spatiotemporal evolution of cubic austenite B2 phase and different variants of monoclinic martensite B19' phase are expressed by twelve continuous field variables $\left\{\Psi_{1}, \Psi_{2}, \cdots \cdots, \Psi_{12}\right\}$ each having values between 0.0 and 1.0. According to the time dependent Ginzburg-Landau (TDGL) phase field kinetic equation, all field variables evolve stochastically over the spatiotemporal domain and the evolution rate is directly proportional to the thermodynamic driving force, i.e.

$$
\frac{\partial \Psi_{i}}{\partial t}=\sum_{j=1}^{12}\left[\widehat{\vartheta}_{i j}\left(\mathrm{G}_{j}^{\text {local }}+\mathrm{G}_{j}^{\text {gradient }}+\mathrm{G}_{j}^{\text {elastic }}\right)\right]+\eta_{i}(\mathbf{x}, t)
$$

where $\Psi_{i}$ represents the $i^{\text {th }}$ martensite variant or the phase field variable, $\widehat{\vartheta}_{i j}$ represents the 
kinetic coefficients matrix, $\mathrm{G}_{j}^{\text {local }} ; \mathrm{G}_{j}^{\text {gradient }}$ and $\mathrm{G}_{j}^{\text {elastic }}$ represent the driving force acting on the $j^{\text {th }}$ field variable due to the local free energy density $g^{\text {local }}$, gradient energy density $g^{\text {gradient }}$ and elastic energy density $g^{\text {elastic }}$, respectively, and $\eta_{i}(\mathbf{x}, t)$ represents the normally distributed and mutually independent (in the spatiotemporal coordinate) Langevin noise term. It is considered that for $i \neq j$, the driving force on the $i^{\text {th }}$ phase field variable does not affect the evolution of $j^{\text {th }}$ phase field variable. Thus, the kinetic coefficients matrix is diagonal i.e. $\widehat{\vartheta}_{i j}=\vartheta \delta_{i j}$, where $\delta_{i j}$ is the Kronecker delta. The requirement for fluctuation-dissipation correlation of this Langevin noise term can be expressed as the function of temperature $\left\langle\eta_{i}(\mathbf{x}, t) \eta_{j}(\mathbf{x}+\mathbf{s}, t+\tau)\right\rangle=2 k_{B} T \widehat{\vartheta}_{i j} \delta_{i j} \delta(\mathbf{s}) \delta(\tau), k_{B}$ is the Boltzmann constant, $\mathrm{T}$ is simulation temperature and $\delta(\cdot)$ the Dirac delta function. Different types for the energy density function and the associated driving force acting on any field variables are considered in the following.

The local free energy density $g^{\text {local }}$ mainly depends on the bulk thermodynamic properties of the system and can be expressed by a Landau type polynomial. Thus, the local free energy density and its associated local driving force are expressed as [31]

$$
\begin{gathered}
g^{\text {local }}=g_{0}+\Delta g(T)\left\{\frac{A}{2}\left(\sum_{i=1}^{12} \Psi_{j}^{2}\right)-\frac{B}{3}\left(\sum_{i=1}^{12} \Psi_{j}^{3}\right)+\frac{C}{4}\left(\sum_{i=1}^{12} \Psi_{j}^{2}\right)^{2}+\frac{D}{4}\left(\sum_{i=1}^{12} \Psi_{j}^{4}\right)\right\} \\
G_{i}^{\text {local }}=-\frac{\partial g^{\text {local }}}{\partial \Psi_{i}}=-\Delta g(T)\left\{A-B \Psi_{i}+C\left(\sum_{j=1}^{12} \Psi_{j}^{2}\right)+D \Psi_{i}^{2}\right\} \Psi_{i}
\end{gathered}
$$

where $g_{0}$ represent the free energy density of the austenite phase, $\Delta g(T)$ the difference in the free energy between austenite and martensite phase at temperature $T$, and $A, B, C$ and $D$ are constants that control the shape of the free energy function. Another important component of the driving force arises from the gradient energy density function $g^{\text {gradient }}$, which is the nonlocal part of the free energy and provides a measure of the interfacial energy between austenite and different martensite twin variants as well as within different martensite twin variants. Thus, the gradient energy density function $g^{\text {gradient }}$ is expressed as [31]

$$
g^{\text {gradient }}=\frac{1}{2} \sum_{i=1}^{12} \beta_{k l}\left(\Psi_{i}\right)\left(\frac{\partial \Psi_{i}}{\partial x_{k}}\right)\left(\frac{\partial \Psi_{i}}{\partial x_{l}}\right)
$$

In (7.a), for isotropic gradient energy, coefficient $\beta_{k l}\left(\Psi_{i}\right)$ can be assumed the same for all field variables, i.e. $\beta_{k l}\left(\Psi_{i}\right)=\beta \delta_{k l}$, and this finally provides the expression for the gradient energy density function and associated driving force as [31]

$$
\begin{gathered}
g^{\text {gradient }}=\frac{1}{2} \sum_{i=1}^{12}\left(\beta \delta_{k l}\right)\left(\frac{\partial \Psi_{i}}{\partial x_{k}}\right)\left(\frac{\partial \Psi_{i}}{\partial x_{l}}\right)=\frac{\beta}{2} \sum_{i=1}^{12}\left(\nabla \Psi_{i}\right)^{2} \\
\mathrm{G}_{i}^{\text {gradient }}=\frac{\partial g^{\text {gradient }}}{\partial \Psi_{i}}=\beta\left(\nabla^{2} \Psi_{i}\right)
\end{gathered}
$$


The non-linear elastic energy density $g^{\text {elastic }}$ can be described through the linearized strain tensor $\varepsilon$, nonlinear stress-free strain due local phase transformation $\varepsilon^{*}$ and isotropic elasticity tensor $\mathbf{C}$, i.e.

$$
g^{\text {elastic }}=\frac{1}{2}\left(\boldsymbol{\varepsilon}-\boldsymbol{\varepsilon}^{*}\right)^{\mathrm{T}} \mathbf{C}\left(\boldsymbol{\varepsilon}-\boldsymbol{\varepsilon}^{*}\right)
$$

where $\boldsymbol{\varepsilon}=\left\{\varepsilon_{11}, \varepsilon_{22}, \varepsilon_{33}, 2 \varepsilon_{23}, 2 \varepsilon_{31}, 2 \varepsilon_{12}\right\}^{\mathrm{T}}$ denotes the total linearized strain. The stress-free strain developed from local phase transformation depends on the evolution of field variables and thus varies in the spatiotemporal domain. It is expressed as $\boldsymbol{\varepsilon}^{*}(\mathbf{x}, t)=\sum_{i=1}^{12} \Psi_{i}(\mathbf{x}, t) \cdot \boldsymbol{\varepsilon}_{i}^{0}$, where $\varepsilon_{i}^{0}$ represents the transformation strain tensor form the $i^{\text {th }}$ variant of martensite B19' phase to austenite B2 phase [31]. In vector notation this stress-free strain tensor is expressed as $\boldsymbol{\varepsilon}^{*}=\left\{\varepsilon_{11}^{*}, \varepsilon_{22}^{*}, \varepsilon_{33}^{*}, 2 \varepsilon_{23}^{*}, 2 \varepsilon_{31}^{*}, 2 \varepsilon_{12}^{*}\right\}^{\mathrm{T}}$. Finally, $\mathbf{C}$ denotes the $6 \times 6$ isotropic elasticity tensor in Voigt notation, and $\mathrm{C}_{11}=183 \mathrm{Gpa} ; \mathrm{C}_{12}=146 \mathrm{Gpa}$ and $\mathrm{C}_{44}=46 \mathrm{Gpa}$ [31]. Therefore, the constitutive equations are expressed as $\boldsymbol{\sigma}=\mathbf{C}\left(\boldsymbol{\varepsilon}-\boldsymbol{\varepsilon}^{*}\right)$; where $\boldsymbol{\sigma}=\left\{\sigma_{11}, \sigma_{22}, \sigma_{33}, \sigma_{23}, \sigma_{31}, \sigma_{12}\right\}^{\mathrm{T}}$. The associated driving force due to the non-linear elastic energy density is express as

$$
\mathrm{G}_{i}^{\text {elastic }}=-\frac{\partial g^{\text {elastic }}}{\partial \Psi_{i}}=\left[\mathbf{C} \cdot\left(\boldsymbol{\varepsilon}-\boldsymbol{\varepsilon}^{*}\right)\right]^{\mathrm{T}} \frac{\partial \boldsymbol{\varepsilon}^{*}}{\partial \Psi_{i}}=\boldsymbol{\sigma}^{\mathrm{T}}\left(\frac{\partial \boldsymbol{\varepsilon}^{*}}{\partial \Psi_{i}}\right)
$$

Elastic interactions play a critical role in the evolution of martensite phase fraction, thus, the accurate estimation of the elastic energy density in Eq. (8.b) is very important. For any $i^{\text {th }}$ variant of martensite B19' phase, at any instant of time and at any spatial coordinate, it is possible to express $\frac{\partial \boldsymbol{\varepsilon}^{*}}{\partial \Psi_{i}}=\boldsymbol{\varepsilon}_{i}^{0}$. Now, in the absence of any external strain tensor $(\varepsilon)$, the constitutive relation can be expressed as $\boldsymbol{\sigma}=-\mathbf{C} \boldsymbol{\varepsilon}^{*}=-\mathbf{C} \sum_{i=1}^{12} \Psi_{i}(\mathbf{x}, t) \cdot \boldsymbol{\varepsilon}_{i}^{0}$. Then, Eq. (8.b) can be expressed as

$$
\mathrm{G}_{i}^{\text {elastic }}=\boldsymbol{\sigma}^{\mathrm{T}}\left(\frac{\partial \boldsymbol{\varepsilon}^{*}}{\partial \Psi_{i}}\right)=-\left[\sum_{i=1}^{12} \Psi_{i}(\mathbf{x}, t) \cdot\left(\boldsymbol{\varepsilon}_{i}^{0}\right)^{\mathrm{T}}\right] \cdot\left(\mathbf{C}^{\mathrm{T}} \boldsymbol{\varepsilon}_{i}^{0}\right)
$$

Once all the terms in TDGL kinetic equation (Eq. (5)) are obtained, the stochastic spatiotemporal evolution of any field variable (e.g. the $i^{\text {th }}$ field variable) can be expressed as

$$
\frac{\partial \Psi_{i}}{\partial t}=\vartheta\left[-\Delta g(T)\left\{A-B \Psi_{i}+C\left(\sum_{j=1}^{12} \Psi_{j}^{2}\right)+D \Psi_{i}^{2}\right\} \Psi_{i}+\beta\left(\nabla^{2} \Psi_{i}\right)+\boldsymbol{\sigma}^{\mathrm{T}}\left(\frac{\partial \mathbf{\varepsilon}^{*}}{\partial \Psi_{i}}\right)\right]+\eta_{i}(\mathbf{x}, t)
$$

Equation (9) is easily normalized in terms of length and time scale. By employing evenly spaced grid size ( $\Delta x)$ and time steps ( $\Delta t)$ (9) yields

$$
\frac{\partial \Psi_{i}}{\partial \tilde{t}}=\left[-\left\{A-B \Psi_{i}+C\left(\sum_{j=1}^{12} \Psi_{j}^{2}\right)+D \Psi_{i}^{2}\right\} \Psi_{i}+\tilde{\beta}\left(\tilde{\nabla}^{2} \Psi_{i}\right)+\boldsymbol{\sigma}^{\mathrm{T}}\left(\frac{\partial \boldsymbol{\varepsilon}^{*} / \partial \Psi_{i}}{\Delta g(T)}\right)\right]+\tilde{\eta}_{i}(\tilde{\mathbf{x}}, \tilde{t})
$$

where dimensionless time and time steps are expressed in terms of real time $t$ and time steps $\Delta t$, i.e. $\tilde{t}=t \vartheta \Delta g(T)$ and $\Delta \tilde{t}=\Delta t \vartheta \Delta g(T)$, and dimensionless spatial coordinate system is 
expressed in terms of grid size $\Delta x$ i.e. $\tilde{\mathbf{x}}=\mathbf{x} / \Delta x$ and $\tilde{\beta}=\tilde{\beta} / \Delta g(T)(\Delta x)^{2}$ denotes the normalized isotropic gradient energy coefficient. Finally, the normalized Langevin noise term $\tilde{\eta}_{i}(\tilde{\mathbf{x}}, \tilde{t})$ is generated as a random number from spatiotemporally uncorrelated normal distribution with zero mean and variance of $2 k_{B} T / \Delta g(T)(\Delta x)^{3}$.

\subsection{Deterministic experimental data at coarse scales}

Zotov et al [43], report extensive coarse scale experimental data on thermally induced martensitic phase fraction. The experiments were performed with $20{ }^{0} \mathrm{C} / \mathrm{min}$ cooling and heating rate. Based on these experimental data on polycrystalline (mainly [110] B2 crystal with some [111] B2 crystal) and annealed (at $750{ }^{\circ} \mathrm{C}$ temperature) NiTi SMA, Zotov et al [43] proposed a phenomenological model and verified it with respect to additional experimental data reported in the literature for NiTi of different concentrations of $\mathrm{Ni}$ and $\mathrm{Ti}[44,45]$. The model captures the kinetics of the observed martensitic phase transformation rate as a function of the martensite phase fraction in the reported experiments adequately. According to the model, the temperature dependent martensite phase fraction evolution is described through the Richards equation, i.e.

$$
\zeta_{c}(T)=1-\left[1+\exp \left\{-g \cdot v\left(T-T_{m}\right)\right\}\right]^{(-1 / v)}
$$

where $\zeta_{c}(T)$ denotes the temperature dependent martensite phase fraction, $T$ the temperature of interest in degrees Kelvin, $T_{m}$ the temperature corresponding to maximum transformation rate in degrees Kelvin, while $g$ (in units of $1 / \mathrm{K}$ ) and $v$ (unitless) are fit parameters which dictate the transformation or growth rate of martensite phases. Equation (11) is adopted here to represent a model for the martensitic phase fraction as a function of temperature at coarse scales. It is noted that in both the MD and $\mathrm{PhF}$ simulations, single crystal pristine $\mathrm{B} 2$ samples are considered. However, the experimental samples are polycrystalline in nature thus contain grain boundaries and interfaces, which add interfacial energy and grain boundary energy to the free energy in single crystal NiTi SMA [34,46]. Even though $\mathrm{PhF}$ simulations for polycrystalline $\mathrm{NiTi}$ are feasible [34], there is a strong interplay between the average grain size in polycrystalline NiTi and the size of the specimen when it comes to thermally induced martensitic phase transformation. Such an interplay is addressed at a subsequent section, and Fig. 7 is provided. To isolate the size effects, single crystal PhF simulations are performed here, and thus MD simulations. It is noted however, that in the polycrystalline NiTi SMA (i.e. in the experimental NiTi SMA sample) martensite start $\left(M_{s}\right)$ and finish $\left(M_{f}\right)$ temperatures are lower than those of the single crystal NiTi SMA, and within the transformation temperature regime (between $M_{s}$ and $M_{f}$ ) the martensitic phase transformation process is enhanced, i.e. the evolution rate of the martensite phase fraction is higher.

\section{Lattice Boltzmann (LB) method for heat transfer}

The Lattice Boltzmann method (LBM) has received extensive attention as a numerical tool in the field of transport phenomena [47-51]. It offers easy application to arbitrary geometry and complex boundaries, significant flexibility such as handling of surface interactions, handling multicomponent systems, domain scalability, and algorithmic parallelizability, which are difficult to invoke through commonly available models based on discretization of the governing differential equations. In LBM, transport of particles and transfer of heat energy 
associated with a particle are performed by tracking movements of particle ensembles or the evolution of the corresponding distribution functions. Particles or its associated energies are initialized on discrete lattice nodes and they move from one lattice node to its neighbors depending on the particle velocity, a step known as propagation. In a following step particles collide and their velocities and energies get updated depending on the equilibrium state of the system.

In two dimensions (2-D), transport can be described by the lattice Boltzmann equation expressed as $[47,50,51]$

$$
f_{\alpha}\left(\mathbf{x}+\mathbf{e}_{\alpha} \delta_{t}, t+\delta_{t}\right)-f_{\alpha}(\mathbf{x}, t)=-\frac{1}{\tau_{v}}\left[f_{\alpha}(\mathbf{x}, t)-f_{\alpha}^{e q}(\rho, \mathbf{u})\right]
$$

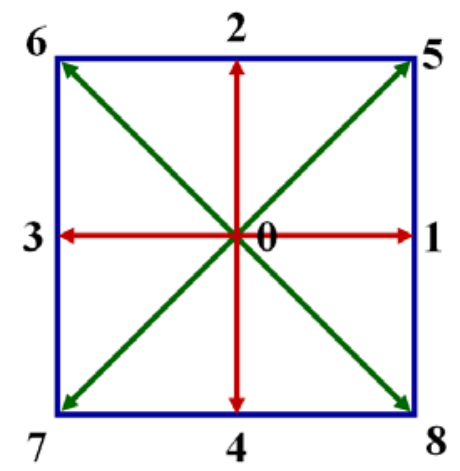

Figure-1: Direction system for D2Q9 LBM lattice configuration, used for particle transport and energy transfer from one grid point to another grid point.

where $f_{\alpha}$ denotes the density distribution function of the particle, $\mathbf{x}$ the space coordinates or position vector, $\mathbf{e}_{a}$ the discrete velocity components, $\mathbf{u}$ the velocity vector, $\delta_{t}$ the time step/ temporal resolution, $\tau_{v}=\frac{3 v}{c \delta_{x}}+\frac{1}{2}$ the dimensionless relaxation time, $v$ the kinematic viscosity of the medium, $\delta_{x}=\delta_{y}$ the lattice constant (grid size)/ spatial resolution, $c$ the speed in the lattice and depends on the spatial and temporal resolution, expressed by $c=\delta_{x} / \delta_{t}$ and this is related to the speed of sound in the lattice through $c_{s}^{2}=R T=\frac{c^{2}}{3}$, where $R$ is the universal gas constant and $T$ is absolute temperature of the system. Here, $f_{\alpha}^{e q}$ denotes the equilibrium distribution of $f_{\alpha}$ and can be expressed through, for the so-called D2Q9 lattice configuration (shown in Fig. 1) [48,50]

$$
f_{\alpha}^{e q}(\rho, \mathbf{u})=\omega_{\alpha} \rho(\mathbf{x}, t)\left[1+3 \frac{\mathbf{e}_{\alpha} \cdot \mathbf{u}}{c^{2}}+9 \frac{\left(\mathbf{e}_{\alpha} \cdot \mathbf{u}\right)^{2}}{2 c^{4}}-\frac{3 \mathbf{u}^{2}}{2 c^{2}}\right]
$$

For the widely used two-dimensional nine-speed (D2Q9) LBM model configuration (as shown in Fig.1), the discrete velocities are expressed as [47,50,52]

$$
\mathbf{e}_{\alpha}=\left\{\begin{array}{cc}
(0,0) & \alpha=0 \\
(\cos [(\alpha-1) \pi / 2], \sin [(\alpha-1) \pi / 2]) c & \alpha=1,2,3,4 \\
\sqrt{2}(\cos [(\alpha-5) \pi / 2+\pi / 4], \sin [(\alpha-5) \pi / 2+\pi / 4]) c & \alpha=5,6,7,8
\end{array}\right.
$$


and the corresponding weight factors/ coefficients are $\omega_{0}=4 / 9, \omega_{\alpha}=1 / 9$ for $\alpha=1,2,3,4$ and $\omega_{\alpha}=1 / 36$ for $\alpha=5,6,7,8$ The density and the velocity are calculated by

$$
\begin{gathered}
\rho(\mathbf{x}, t)=\sum_{\alpha} f_{\alpha}(\mathbf{x}, t) \\
\mathbf{u}=\sum_{\alpha} \mathbf{e}_{\boldsymbol{\alpha}} f_{\alpha}(\mathbf{x}, t) / \rho(\mathbf{x}, t)
\end{gathered}
$$

Next, to model the heat transfer process, it is assumed that heat diffuses through the NiTi medium and heat energy is dissipated due to the phase transformation process. Thus, for the transient case, the generalized heat diffusion equation can be expressed as

$$
\frac{d T}{d t}=\left(\frac{1}{\rho c_{p}}\right) \nabla \cdot(k \nabla \cdot T)-\left(\frac{\dot{Q}}{\rho c_{p}}\right)
$$

Now, the LBM formulation for heat energy transfer through diffusion (i.e. Eq. 16.a) for the D2Q9 configuration is presented. Considering an isothermal process for heat transfer, Peng's implementation of thermal LBM [53] is a simplified form of He et al [54] thermal LBM. Here, the heat transfer evolution function with heat source is express as

$$
g_{\alpha}\left(\mathbf{x}+\mathbf{e}_{\alpha} \delta_{t}, t+\delta_{t}\right)-g_{\alpha}(\mathbf{x}, t)=-\frac{1}{\tau_{g}}\left[g_{\alpha}(\mathbf{x}, t)-g_{\alpha}^{e q}(T, \mathbf{u})\right]+\varpi_{\alpha}\left(1-\frac{0.5}{\tau_{g}}\right) \frac{\dot{Q}}{\rho c_{p}}
$$

where $g_{\alpha}$ denotes the heat energy distribution function, $\tau_{g}=\frac{3}{2} \frac{k}{c \delta_{x} \rho c_{p}}+\frac{1}{2}$ the dimensionless relaxation time for heat energy transfer, $\rho, k, c_{p}$ and $\dot{Q}$ the density, thermal conductivity, heat capacity and heat energy dissipation respectively, measured at any lattice node and any instant of time. For this study, the temperature dependent thermal conductivity and heat capacity parameters are obtained from different experimental studies [55-57]. For the D2Q9 lattice system, the equilibrium distribution function of heat energy is expressed as [58,59]

$$
g_{\alpha}^{e q}\left(c_{i}, \mathbf{u}\right)=\left\{\begin{array}{cc}
-\varpi_{\alpha} T(\mathbf{x}, t) \frac{\mathbf{u}^{2}}{c^{2}} & \alpha=0 \\
\varpi_{\alpha} T(\mathbf{x}, t)\left[\frac{3}{2}+\frac{3}{2} \frac{\mathbf{e}_{\alpha} \cdot \mathbf{u}}{c^{2}}+\frac{9}{2} \frac{\left(\mathbf{e}_{\boldsymbol{\alpha}} \cdot \mathbf{u}\right)^{2}}{c^{4}}-\frac{3}{2} \frac{\mathbf{u}^{2}}{c^{2}}\right] & \alpha=1,2,3,4 \\
\varpi_{\alpha} T(\mathbf{x}, t)\left[3+6 \frac{\mathbf{e}_{\boldsymbol{\alpha}} \cdot \mathbf{u}}{c^{2}}+\frac{9}{2} \frac{\left(\mathbf{e}_{\boldsymbol{\alpha}} \cdot \mathbf{u}\right)^{2}}{c^{4}}-\frac{3}{2} \frac{\mathbf{u}^{2}}{c^{2}}\right] & \alpha=5,6,7,8
\end{array}\right.
$$

For heat energy transfer the discrete velocities are those in Eq. (14), with weight factors $\varpi_{0}=-2 / 3, \varpi_{\alpha}=1 / 9$ for $\alpha=1,2,3,4$ and $\varpi_{\alpha}=1 / 36$ for $\alpha=5,6,7,8$. The temperature is calculated by

$$
T(\mathbf{x}, t)=\sum_{\alpha} g_{\alpha}(\mathbf{x}, t)+\frac{\delta_{t}}{2} \frac{\dot{Q}}{\rho c_{p}}
$$

In the LBM for heat transfer through a solid all velocity components are zero i.e. $\mathbf{u}=0$. 


\section{Wavelets as multiscale interface}

Wavelet analysis serves as a valuable tool for numerical analysis, data processing, signal processing, and image processing. Wavelets analysis can be conceived as a "mathematical microscope", which easily distinguishes the broader features of say a signal at coarse scales from the fine features at fine scales. The inherent capability of the wavelets to provide scale specific resolution makes it an alternative choice to apply in the field of localized feature extraction from irregular/ non-stationary data. Since the theory and motivation for wavelet analysis are already widely covered in the literature, we do not repeat those details here, but simply provide a brief overview and refer the reader to the following major references on the subject $[5-9,14,22]$. In this study an important predictive feature is added to the compound wavelet matrix (CWM) method. This predictive feature is mainly based on the recent study by Gur et al [60] on the wavelet based surrogate time-series generation for heterogeneous catalysis. The following section describes the CWM methodology.

\subsection{The CWM}

In 1-D (extendable to higher dimensions), the wavelet transform $W_{f}(a, b)$ of a fluctuating function $f(x)$ is expressed as

$$
W_{f}(a, b)=\int_{-\infty}^{\infty} f(x) \psi_{a, b}(x) d x
$$

where the two-parameter family of function $\psi_{a, b}(x)=(1 / \sqrt{a}) \psi[(x-b) / a]$ is obtained from a single mother wavelet function $\psi(x)$ through the dilatations by scaling factor $a$ and translation by the factor $b$. The factor $1 / \sqrt{a}$ is included for normalization, such that the energy of the wavelet at each scale remains the same. The parameter $a$ can take any possible positive real value, and the fluctuations of $f(x)$ are measured at position $b$, and at scale $a$. If $f(x)$ is only available at specific and even slightly overlapping wavelet space scales, say for simplicity at fine, $a_{\text {fine }}$ and coarse, $a_{\text {coarse }}$ scales, then the wavelet synthesis or fusion process implies, in a statistical sense,

$$
W_{f}(a, b)=W_{f}\left(a_{\text {fine }}, b\right) \cup W_{f}\left(a_{\text {coarse }}, b\right)
$$

i.e. the union (with proper weights for overlapping scales) of the wavelet transform at fine and coarse scales. Properties of $f(x)$ such as statistical moments can be determined via the inverse wavelet transform (IWT), thus for example the mean value of $f(x),\langle f(x)\rangle$ is expressed as

$$
\langle f(x)\rangle=\left\langle\operatorname{IWT}\left[W_{f}\left(a_{\text {fine }}, b\right) \cup W_{f}\left(a_{\text {coarse }}, b\right)\right]\right\rangle
$$

and corresponding relations hold for higher statistical moments and spatial correlations. The above forms the essentials of the CWM multiscale method.

\subsection{Wavelet based multiscaling interface: $C W M$}

In a wavelet transform process, a 1-D discrete data vector of length $2^{N}$ is hierarchically decomposed into $N$ scales and the transform returns $2^{N}$ wavelet transform (WT) coefficients. The first $2^{N-1}$ WT coefficients represent the finest characteristics of the original signal, the 
next $2^{N-2}$ WT coefficients represent the next level (coarser) information in the data and so on. This process is well documented in the literature $[4,5,14]$.

To describe the wavelet-based multiscaling interface in the martensitic phase transformation process, a 1-D example is considered and discussed. For thermally induced martensitic phase transformation, the martensite phase fraction at any grid point of the phase field is represented by the vector $\{\xi\}_{f}$ and through the wavelet interface it can be coupled to martensite phase fraction at any node of deterministic simulation as denoted by the vector $\{\zeta\}_{c}$, and this will finally yield a (coupled) martensite phase fraction vector $\{\varsigma\}_{c w m}$. Here, $f$ stands for the fine simulation (phase field) process and the number of data points resulting from the phase field simulation is $n_{f}$ with the grid spacing $\Delta x$. Similarly, $c$ stands for the coarse data (which can be the result of coarse scale simulations, yet for the present work is experimental data) and the number of coarse scale data points is $n_{c}$ within a grid spacing $\Delta X$ which is typically much larger that fine simulation grid spacing $\Delta x$, i.e. $\Delta X>\Delta x$; for the present case the coarse grid spacing is equal to the size of the studied cell. To par the grid spacing the coarse scale data is discretized to the small scale one and this results into a larger data set with a new spacing $\Delta x$ and the number of data points $N_{c}=n_{c}(\Delta X / \Delta x)$. The wavelet transform of $\{\xi\}_{f}$ will yield $W_{\xi}(s, x)$ i.e. a set of wavelet coefficients, where $s$ represents scale and $x$ stands for spatial coordinate in the fine scale simulation. Now, $W_{\xi}(s, x)$ is decomposed as

$$
W_{\xi}(s, x)=\phi_{\xi}\left(s_{0}, \Delta x\right) \oplus \phi_{\xi}\left(s_{1}, \Delta x\right) \oplus \phi_{\xi}\left(s_{2}, 2^{1} \Delta x\right) \oplus \cdots \cdots \oplus \phi_{\xi}\left(s_{p_{f}}, 2^{p_{f}-1} \Delta x\right)
$$

i.e. into $p_{f}=\log _{2}\left(n_{f}\right)-2^{2}$ number of scales, where $\oplus$ denotes scale wise association in the wavelet formalism, $\phi_{\xi}\left(s_{i}, 2^{i-1} \Delta x\right) i=1,2, \cdots, p_{f}$ denotes the wavelet transform at scale $s_{i}$ with the scaling interval $2^{i-1} \Delta x$ and $\phi_{\xi}\left(s_{0}, \Delta x\right)$ represents the wavelet transform at the coarsest scale in fine scale simulation for a particular scaling function. Similarly, the wavelet transform of $\{\zeta\}_{c}$ yields a set of wavelet coefficient $W_{\zeta}(s, x)$ which is decomposed as

$$
W_{\zeta}(s, x)=\Phi_{\zeta}\left(s_{0}, \Delta x\right) \oplus \Phi_{\zeta}\left(s_{1}, \Delta x\right) \oplus \Phi_{\zeta}\left(s_{2}, 2^{1} \Delta x\right) \oplus \cdots \cdots \oplus \Phi_{\zeta}\left(s_{p_{c}}, 2^{p_{c}-1} \Delta x\right)
$$

i.e. into $p_{c}=\log _{2}\left(N_{c}\right)-2^{2}$ number of scales, where $\Phi_{\zeta}\left(s_{i}, 2^{i-1} \Delta x\right) i=1,2, \cdots, p_{c}$ denotes the wavelet transform at scale $S_{i}$ with the scaling interval $2^{i-1} \Delta x$ and $\Phi_{\zeta}\left(s_{0}, \Delta x\right)$ denotes the wavelet transform at the coarsest scale in deterministic simulation for a particular scaling function.

Once the wavelet coefficient at each scale are obtained (here from phase field simulation and deterministic experimental data) they are coupled via the CWM method. Since the total number of available scales from the fine scale phase field simulation is less than the total number of available scales from the coarse data, i.e. $N>n$, the maximum number of scales that can be coupled is $n$. However, in the CWM method instead of adopting all of the $n$ fine simulation scales, a cutoff scale $\left(n_{\text {cut }}\right)$ is considered based on the fractional energy index $\left(\eta=\sum_{i=1}^{m \leq M} E_{i} / E\right)$ 
, where, $E\left(=\sum_{i=1}^{M} E_{i}\right)$ is the total energy from all scales, $E_{i}$ of the energy of the $i^{\text {th }}$ scale, $m$ the numbers of scales considered and $M$ the total numbers of scales and equal to $n$ for the phase field simulations and $N$ for the coarse data. The value of the cutoff scale $\left(n_{\text {cut }}\right)$ depends on the distribution of the energy of the wavelet transform and choosing a proper value involves scientific/engineering reasoning. Next, the wavelet coefficients from the phase field and deterministic simulation are coupled. To construct the compounded wavelet transform of the martensite phase fraction vector all wavelet coefficients up to the cutoff scale $\left(n_{\text {cut }}\right)$ are directly adopted from the wavelet transform of the coarse data. The CWM method or each iteration in the dynamic CWM considers that scale-wise fluctuations are stationary or quasi-stationary. Then, at all scales above the cutoff scale $\left(n_{\text {cut }}\right)$, the wavelet coefficients are those from the fine simulation which are repeated periodically [6], a process that is valid when the process is stationary or quasi stationary. However, when there are long range correlations in the process (such as the martensitic phase transformation, where fluctuations present in the fine simulation are correlated colored noise) repetitions should be restrained over small scales. In the present study an appropriate scale wise prediction process for the wavelet coefficients above the cutoff scale from fine simulation is considered as proposed in Gur et al [60]. Above the cutoff scale the wavelet coefficients are predicted based on the mean, standard deviation and other higher order statistical moments such as skewness and kurtosis. For any particular scale, using the K$S$ test (Kolmogorov-Smirnov test) from the available wavelet coefficients the type of distribution of the coefficients results. Then, random wavelet coefficients for that particular scale following the identified distribution are created.

The process described above yields sets of wavelets coefficients that contain information at all scales, i.e. the compound scales from the fine and coarse scale data. The process and can expressed mathematically as

$$
\begin{aligned}
W_{\zeta}(s, x)= & \left\{\bar{W}_{\zeta}(s, x)\right\} \oplus\left\{\tilde{W}_{\xi}(s, x)\right\} \\
= & \left\{\Phi_{\zeta}\left(s_{0}, \Delta x\right) \oplus \Phi_{\zeta}\left(s_{1}, \Delta x\right) \oplus \Phi_{\zeta}\left(s_{2}, 2^{1} \Delta x\right) \oplus \cdots \cdots \oplus \Phi_{\zeta}\left(s_{n_{c u t}}, 2^{n_{c u t}} \Delta x\right)\right\} \\
& \oplus\left\{\tilde{\phi}_{\xi}\left(s_{n_{\text {cut }}+1}, 2^{n_{\text {cut }}+1} \Delta x\right) \oplus \tilde{\phi}_{\xi}\left(s_{n_{\text {cut }}+2}, 2^{n_{\text {cut }}+2} \Delta x\right) \oplus \cdots \cdots \oplus \widetilde{\phi}_{\xi}\left(s_{p_{c}}, 2^{p_{c}-1} \Delta x\right)\right\}
\end{aligned}
$$

where $\bar{W}_{\zeta}(s, x)$ represents the wavelet coefficients from the coarse scale data up to the cutoff scale and $\tilde{W}_{\xi}(s, x)$ represents the randomized wavelet coefficient above the cutoff scale $\left(n_{\text {cut }}\right)$ from fine scale simulation (phase field). The inverse/ back wavelet transform is now performed on the compounded wavelet coefficients, i.e. on $W_{s}(s, x)$ to obtain the martensite phase fraction vector after compounding, thus

$$
\{\varsigma\}_{c w m}=W_{\varsigma}^{-1}\left[\varsigma\left\{W_{\varsigma}(s, x)\right\}\right]=W_{\varsigma}^{-1}\left[\varsigma\left(\left\{\bar{W}_{\zeta}(s, x)\right\} \oplus\left\{\tilde{W}_{\xi}(s, x)\right\}\right)\right]
$$

The CWM multiscaling process was described above for simplicity in 1-D, yet its extension to 2-D is straightforward. In the 2-D case, both coarse and fine scale simulations are performed in 2-D, and so is the forward and inverse wavelet transform. The relevant flow chart for the CWM method with the component for predicting the fine scale wavelet coefficients has been provided in the supplementary material.

\section{Results and discussions}


Figure 2 shows the thermally induced martensite phase fraction at small scales as obtained from $\mathrm{PhF}$ stochastic simulations and at the macro scale as obtained from continuum level experimental measurements. Since $\mathrm{PhF}$ simulation is stochastic in nature, the developed martensite phase fraction has built-in uncertainties, as shown in Figure 2 through the "error bars”. The continuum experiments are deterministic and thus no uncertainties are present, as also shown in Figure 2 Phase field simulations were performed on a 2-D, $1024 \mathrm{~nm} \times 1024 \mathrm{~nm}$ NiTi sample size, whereas for the continuum case martensitic phase fraction values at different temperatures were obtained from the study by Zotov et al [43] which is based on experimental measurements.

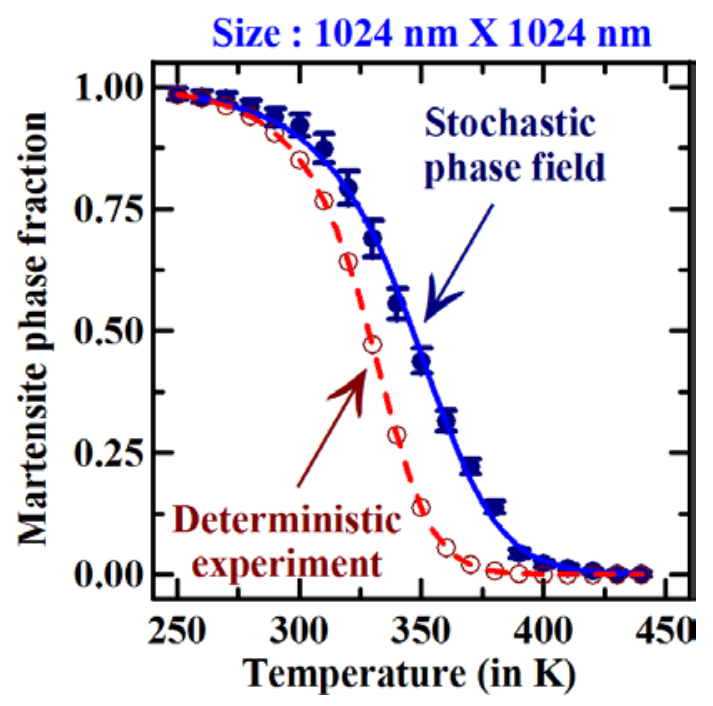

Figure-2: Variation of martensite phase fraction with respect to temperature from phase field simulation (stochastic) and deterministic experiments.

The difference in martensite phase fraction between the stochastic phase field simulation results and deterministic experimental data, within the transformation temperature zone ( 250 - $450 \mathrm{~K}$ ), can be observed, becoming maximum near $350 \mathrm{~K}$. This difference is mainly due to size effects and consideration of the single crystal (in the simulations) vs. the polycrystalline (in the experiment) NiTi SMA samples; this is addressed in detail in the following sections. Significant size effects have been reported extensively, [25,27,28,30,61] where it is also demonstrated that the presence of defects such as free surfaces can appreciably alter the martensite transformation start $\left(M_{s}\right)$ and finish $\left(M_{f}\right)$ temperature ranges as well as the martensite phase fraction value at different temperatures within the transformation temperature zone, i.e. between $M_{s}$ and $M_{f}$.

Even though the primary objective of this work is to implement and demonstrate multiscale modeling of phase fraction, relevant size effects are important and provide a link between and verification of the different models used at different scales. For that reason, in addition to the phase field and continuum based models, molecular dynamics (MD) simulations at small scales are also performed. To study the size effects of the simulation cell, thin films of different sizes (side length from $2-128 \mathrm{~nm}$ ) are considered, with their thickness being equal to $2 \mathrm{~nm}$. In the simulations, initially single B2 crystal with crystallographic orientation [110], [110] and [001] are considered and then the simulation cell is stabilized as mentioned in section 2. For studying size effects, free surface boundary conditions in all directions ( $\mathrm{x}, \mathrm{y}$ and $\mathrm{z}$ ) are considered. MD 
simulation (stabilization) is performed at constant temperature and the martensite phase fraction is evaluated.

Size effects are demonstrated in Figure 3 and some relevant results (evolution of microstructure at different temperatures and sizes) are documented in the supplementary material. Figure 3 shows the variation of martensite phase fraction with respect to temperature for different size of NiTi films. Within the transformation temperature zone (here in MD simulation, considering all the sizes, it is taken to be within the temperature range of $300-550 \mathrm{~K}$ ) prominent size effects can be observed, whereas outside the transformation temperature zone size effects diminish. Because of the size effects, a systematic shift in the transformation temperature regime (i.e. martensite start $\left(M_{s}\right)$ and finish $\left(M_{f}\right)$ temperature) is observed, which shows a decreasing trend with increasing size. The size effects at the atomic level can be attributed to the available free surface area per unit volume and the associated free surface energy. The available free surface area per unit volume of a NiTi thin film is inversely proportional to its side length $s$ i.e. $A / V=\gamma \propto 1 / s$ and with the increase in size this ratio decreases fast, i.e. $d(A / V) / d s=d \gamma / d s \propto-1 / s^{2}$. The available free surface area per unit volume can be linked to the free surface energy per unit volume for transforming the austenite (B2) in to the different martensite B19' variants. Therefore, with decreasing size more free surface energy is available (as the free surface area per unit volume increases) for martensitic phase transformation.

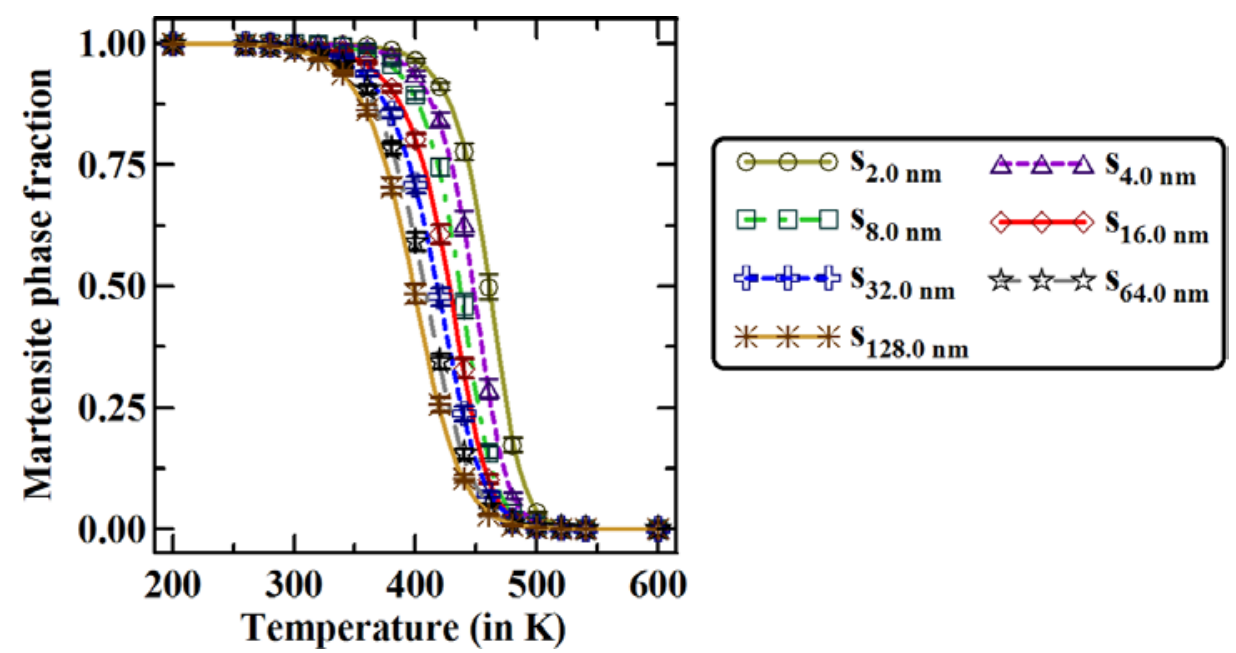

Figure-3: Variation of the martensite phase fraction with respect to temperature resulting from MD simulations for different size of MD simulation cells (thin films).

Similarly to the fine scales (i.e. MD simulations), to demonstrate the size effect at meso scales 2-D thin films of B 2 single crystal are considered and then the simulation cell is stabilized as mentioned in section 2. Here also the crystallographic orientation of the B 2 crystal are [110] and [001]. Size effects at meso scales are demonstrated in Figure 4, where it can be observed that with increasing size the thermally induced martensite phase fraction is decreasing, and, according to Figure 2, it finally converges to the deterministic experimental results. Further, within the transformation temperature regime (in stochastic PF simulation, considering all the sizes, it is within the temperature range of $250-500 \mathrm{~K}$ ) pronounced size effects can be observed, whereas outside the transformation temperature regime size effects die out. Interestingly, the phase field simulation results for different sizes of films also show a 
systematic shift in the martensite start $\left(M_{s}\right)$ and finish $\left(M_{f}\right)$ temperatures, which was also noticed in the MD simulations.

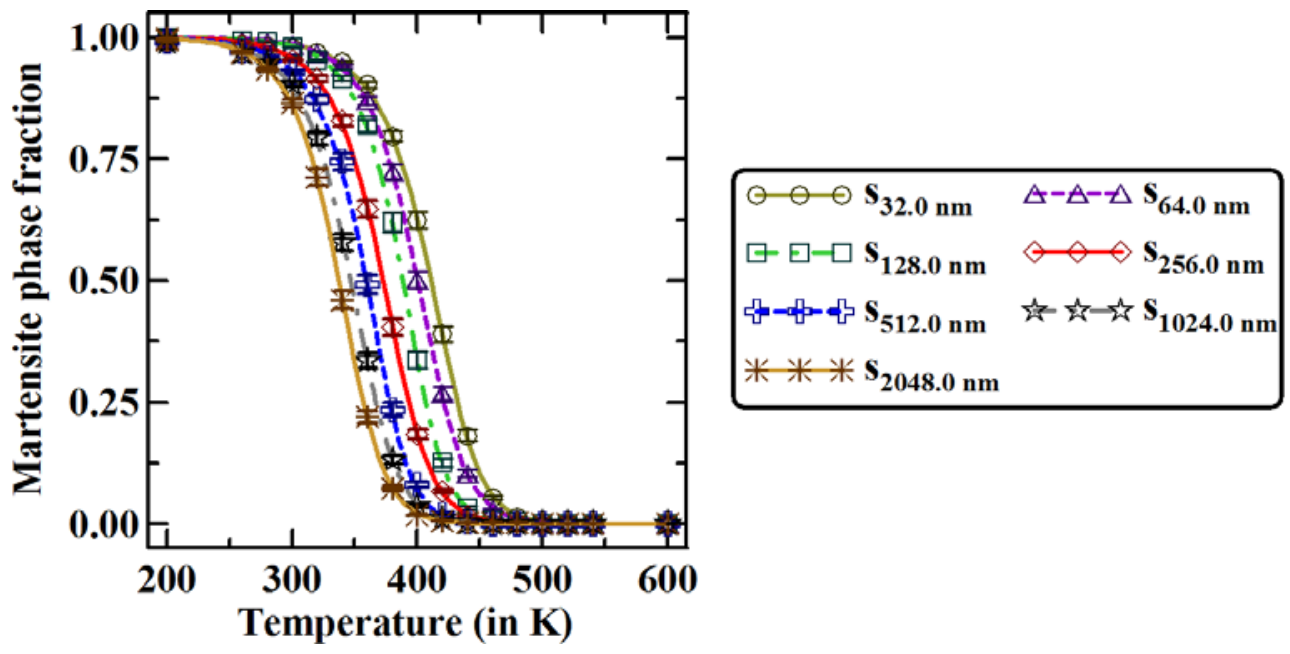

Figure-4: (a) Variation of the martensite phase fraction with respect to temperature resulting from the phase field (PhF) simulation, for different size of PhF simulation cells (thin films).

The influence of size effects on the thermally induced martensitic phase transformation process in NiTi SMA is now examined. As discussed already, within the transformation temperature zone, noticeable size effects on the evolution kinetics of martensite phase fraction are observed for scales ranging from atomic (micro) to meso.

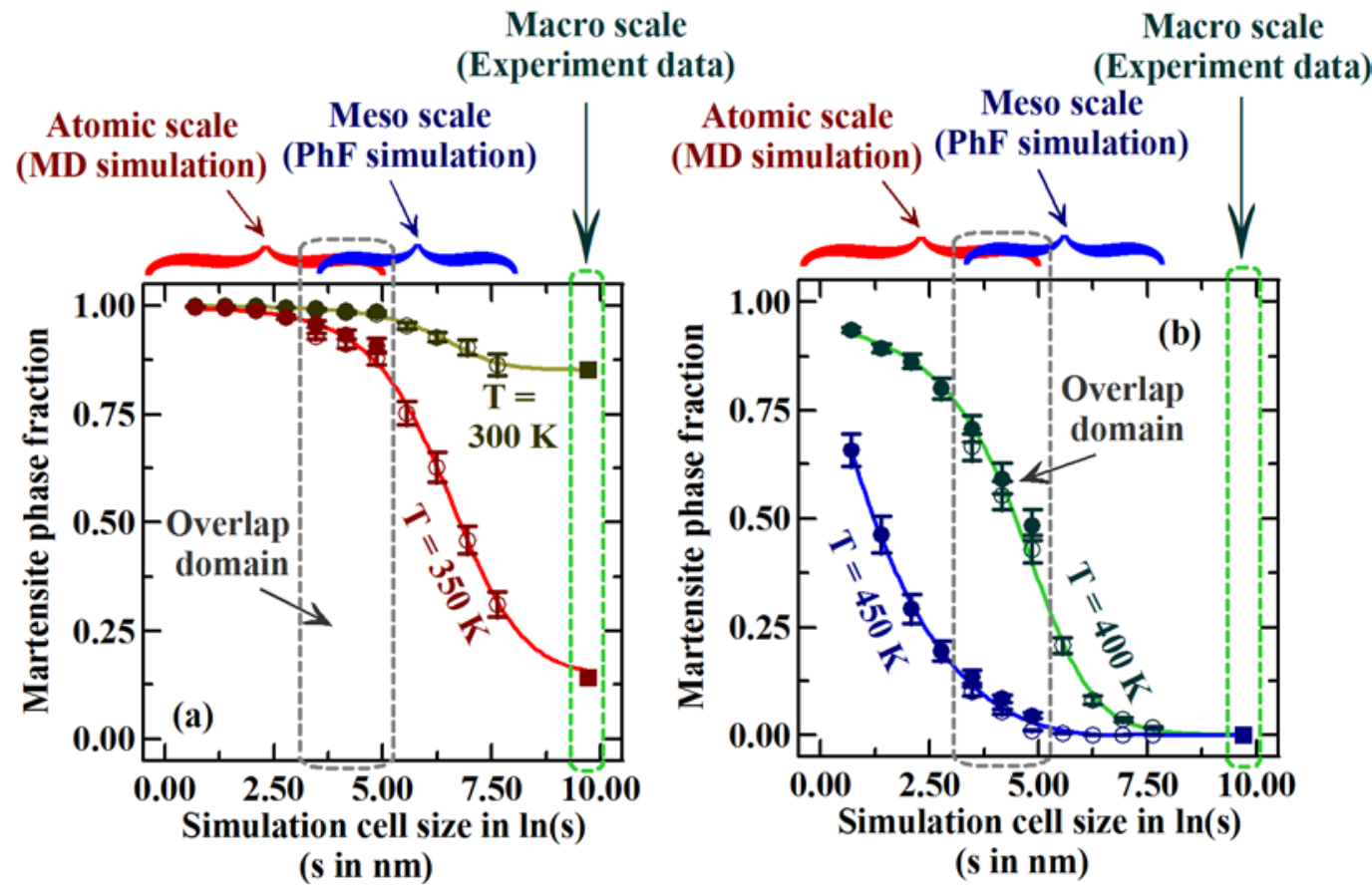

Figure-5: Variation of the martensite phase fraction with respect to simulation cell size at (a) $\mathrm{T}=300 \mathrm{~K}$ and $\mathrm{T}=350 \mathrm{~K}$, (b) $\mathrm{T}=400 \mathrm{~K}$ and $\mathrm{T}=450 \mathrm{~K}$ based on different data (from $\mathrm{MD}$, $\mathrm{PhF}$, and experimental). Solid circles correspond to MD, empty circles to PhF and solid rectangles to deterministic experimental data [43]. There are overlapping MD and PhF scales indicated as “overlap domain”. Due to the scale disparity the cell size axis is logarithmic. 
Figure 5 demonstrates the size effects, where, for brevity, only the evolution of martensite phase fraction with respect to size is shown at select temperatures of interest. However, size effects are observed within the overall transformation temperature regime, $250-550 \mathrm{~K}$. Three cell sizes ( $32 \mathrm{~nm}, 64 \mathrm{~nm}$ and $128 \mathrm{~nm}$ ) are within the overlapping domain between MD and PF simulations where the two methods show very good agreement with each other given the inherently stochastic nature of the thermally induced martensitic phase transformation process. Given the disparity in scales, (from $2 \mathrm{~nm}$ to $16384 \mathrm{~nm}$ ), log scale is used for the cell size axis.

To verify the PhF results from the MD simulations, as well as to quantify the effect of simulation cell size on the uncertainties of the martensite phase fraction evolution, the standard deviation of the martensite phase fraction obtained from MD is compared to that from $\mathrm{PhF}$. Figure 6 shows relevant results for various SMA film sizes and temperatures, where the MD, PhF overlap domain includes film sizes of $32 \mathrm{~nm}, 64 \mathrm{~nm}$ and $128 \mathrm{~nm}$. It is clearly noticeable from Fig. $6 \mathrm{~b}$ that, within the overlap domain, the standard deviation values of martensite phase fraction agree very well with each other. Therefore, it can be expected that the statistics of the martensite phase fraction evolution will be transferred from the PhF scales to the macroscopic ones appropriately.
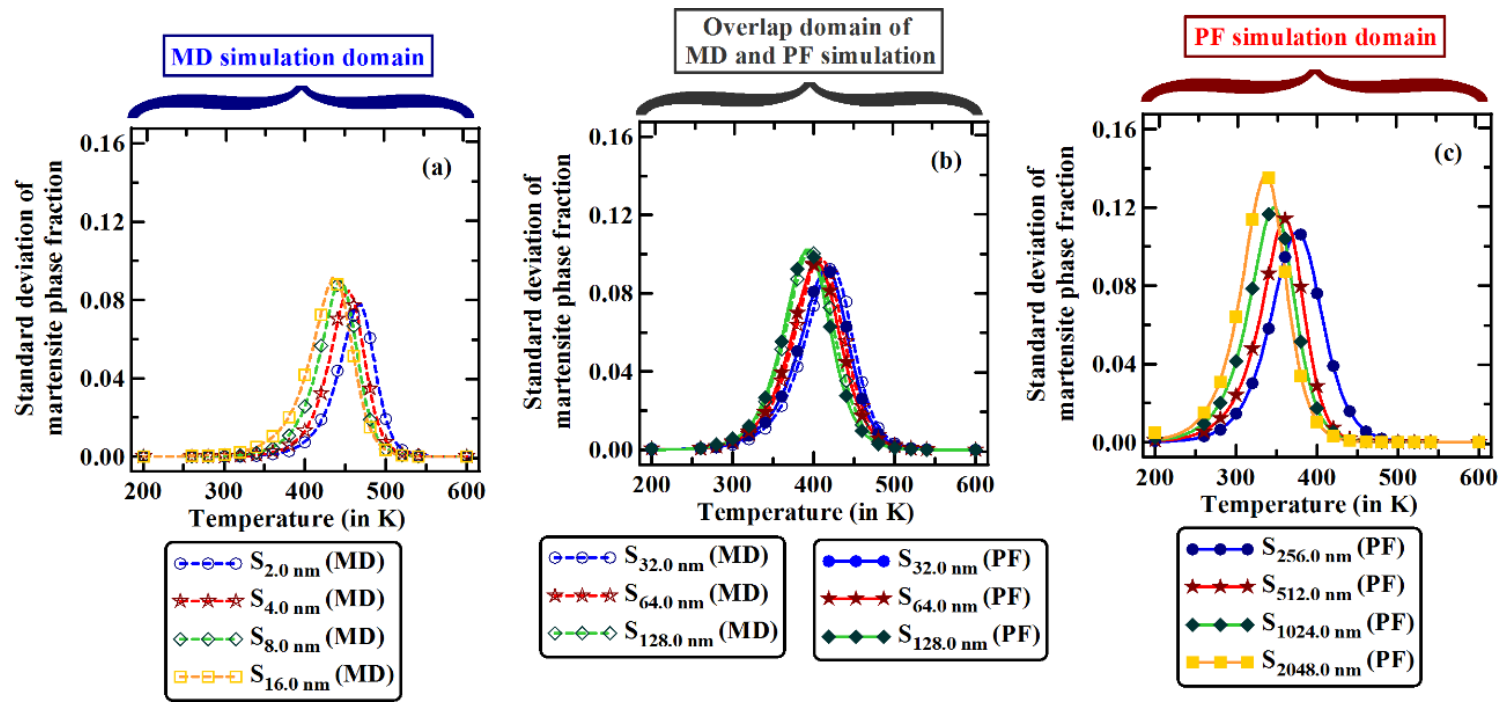

Figure-6: Variation of the standard deviation of the martensite phase fraction with respect to the temperature for different size from different simulation methods (a) MD simulation domain, (b) Overlap simulation domain of MD and PhF and (c) PhF simulation domain.

Finally, before moving to the multiscale modeling aspect, the interplay between grain size and cell size in polycrystalline NiTi is discussed with respect to temperature induced martensitic transformation. As mentioned before, in this work in order to isolate the size effects from the grain size effects, single crystal configurations were simulated. Figure 7 shows the effects of grain size on martensitic phase fraction at a fixed temperature $\mathrm{T}=313 \mathrm{~K}$ as well as the effects of cell size for single NiTi crystals. In the experimental data reported in [43] the average grain size is $44.72 \mathrm{~nm}$. In Fig. 7a the simulation cell size in the reported PhF simulations [34] is 512 $\mathrm{nm}$, thus the phase fraction for the single crystal simulations is pointed in Fig. 7b. The interplay of cell/specimen size and grain size, despite its importance, has received little attention, perhaps because of the difficulties in addressing it. Some attempts to shed light in such interplay are reported in [62] albeit for mechanical properties. 

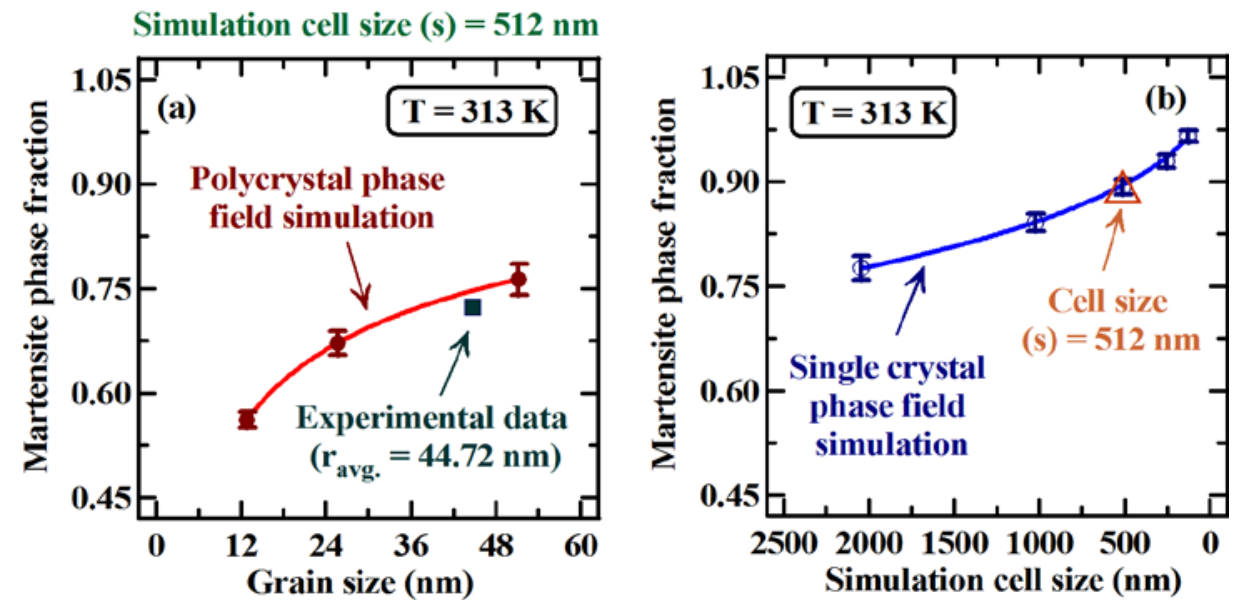

Figure-7: Single crystal versus polycrystalline results. (a) Grain size effect on martensitic phase fraction at a fixed temperature $\mathrm{T}=313 \mathrm{~K}$ based on existing PhF simulations [34] and on available experimental data [43]; (b) Size effect on martensitic phase fraction at a fixed temperature $\mathrm{T}=313 \mathrm{~K}$ based on single crystal $\mathrm{PhF}$ simulations.

From this study, and from the other studies at the micro scale (mainly employing MD simulations), it is clear that there exist predominant size effects starting from micro scale (atomistic level) up to the macro scale (continuum level). From the seven different cell sizes examined, that of size $1024 \mathrm{~nm} \times 1024 \mathrm{~nm}$ is approaching the converged size regime (in terms of the martensite phase fraction with respect to the cell size) and thus is chosen to be coupled to experiment-based results on considered cell size of $16384 \mathrm{~nm} \times 16384 \mathrm{~nm}$. The coupling is performed via the CWM methodology. Three distinct temperatures are considered, i.e. $\mathrm{T}=320 \mathrm{~K}$ (where mostly the sample is martensite), $\mathrm{T}=350 \mathrm{~K}$ (where the sample is about half martensite half austenite), and $\mathrm{T}=380 \mathrm{~K}$ (where the sample is mostly austenite) and different statistical measures (mean, standard deviation and other higher order moments such as skewness and kurtosis) of the martensite phase fraction evolution are determined. Further, the spatial autocorrelation function of the martensitic phase fraction is evaluated. All statistical measures as obtained from CWM are compared to those obtained from $\mathrm{PhF}$ and when possible to the deterministic experimental data.

Table-1: Statistical measures of martensite phase fraction at different temperatures obtained from $\mathrm{PhF}$ (with residual size effects and for single crystals), deterministic experimental data and from CWM method (i.e. after multiscale coupling)

\begin{tabular}{ccccc}
\hline \hline \multicolumn{2}{c}{ Temperature (in K) } & $\mathrm{T}=320$ & $\mathrm{~T}=350$ & $\mathrm{~T}=380$ \\
\hline \hline \multirow{3}{*}{ Mean } & Phase Field & 0.794 & 0.438 & 0.138 \\
\cline { 2 - 5 } & Experimental Data & 0.659 & 0.148 & 0.017 \\
\cline { 2 - 5 } Standard & CWM & 0.661 & 0.157 & 0.023 \\
\hline \multirow{2}{*}{ Skeviation } & Phase Field & 0.097 & 0.073 & 0.033 \\
\hline \multirow{2}{*}{ Kurtosis } & CWM & 0.101 & 0.078 & 0.032 \\
\hline \multirow{2}{*}{$\begin{array}{c}\text { Correlation } \\
\text { distance }(\mathrm{in} \mathrm{nm})\end{array}$} & Phase Field & 0.798 & 0.950 & 0.330 \\
\hline \hline
\end{tabular}


Table- 1 shows the relevant measures. Of course, since the experimental (coarse scale) values of martensite phase fraction are deterministic, the comparison is only valid with respect to mean values. In terms of mean value of the martensite phase fraction, the CWM results match very well with the experimental data but the residual size effects show a difference with the PhF simulation results. Also, these difference in the phase field simulation results and experimental data of the mean value of martensite phase fraction are due to the consideration of single crystal in phase field simulation, whereas experimental samples are polycrystalline. In terms of other statistical measures, the CWM results match very well the $\mathrm{PhF}$ ones. It can be concluded that the CWM method accurately captures and transfers the material related uncertainties as well as spatial statistical information from fine/ meso to the coarse scale, while at the same time agrees with the martensite phase fraction evolution at coarse scales. Some of the PhF and multiscale coupling results are shown next, and further details are provided in the supplementary material. Figures 8 (a1) and (a2) show the spatial evolution of martensitic phase fraction (fluctuating part). Figures 8 (b1) and (b2) show the autocorrelation function from PhF simulation and after performing the CWM process at $\mathrm{T}=350 \mathrm{~K}$. It can be observed that the spatial fluctuations of martensitic phase fraction, including the auto correlation functions, obtained from PhF simulation agree well with those obtained from CWM.
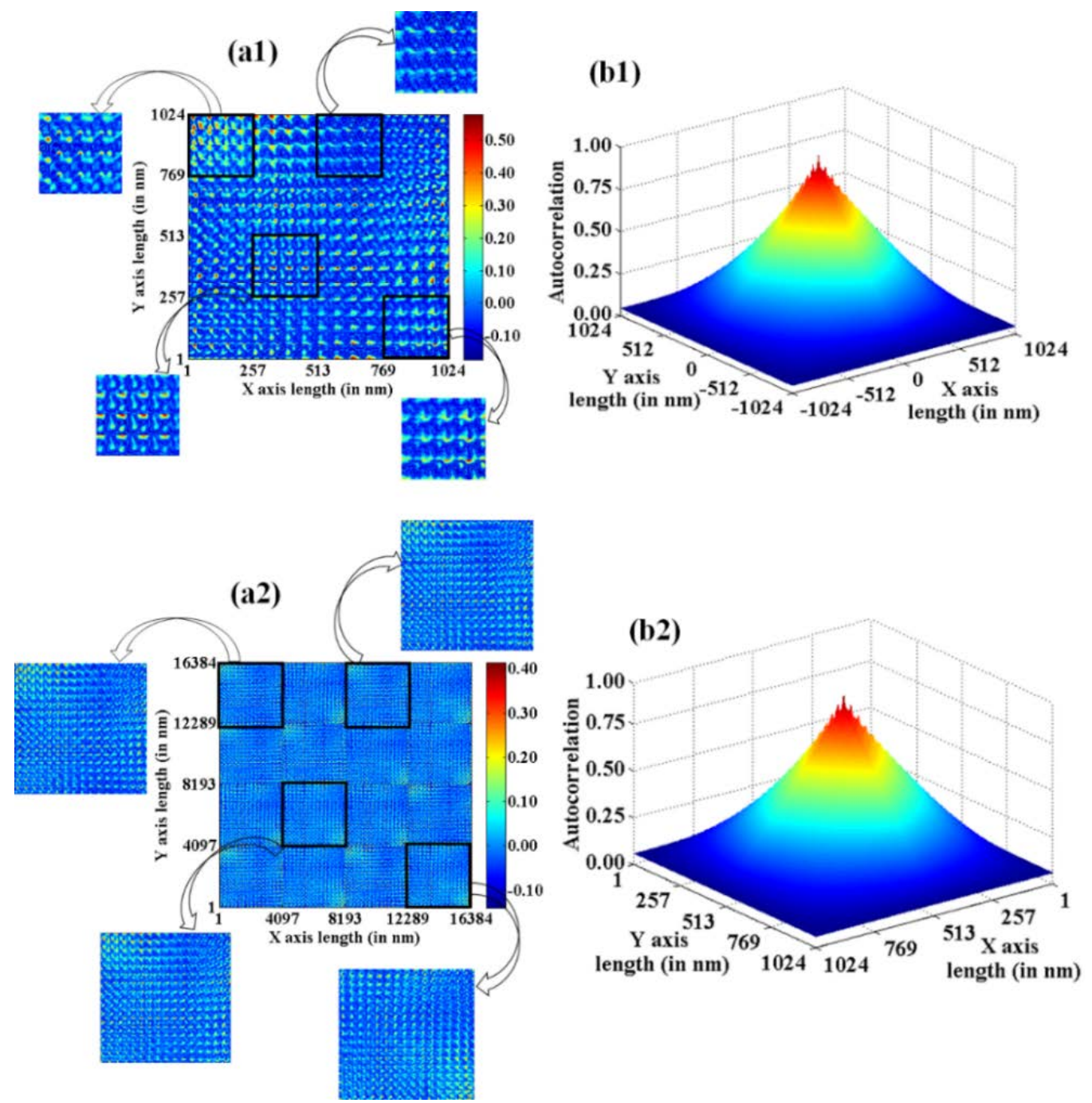

Figure-8: Spatial pattern of the fluctuating part of martensite phase fraction at $\mathrm{T}=350 \mathrm{~K}$ from (a1) PhF and (a2) after coupling via the CWM method. Autocorrelation function of martensite 
phase fraction at $\mathrm{T}=350 \mathrm{~K}$ from (b1) phase field simulation and (b2) after coupling via CWM method.

Next, the evolution of different B19' variants is shown. It is well known that 3-D NiTi SMA simulation cells are capable of accommodating all 12 B19' variants. However, in 2-D NiTi simulation cells, due to the absence of third direction all 12 B19' variants are not be able to evolve simultaneously [34,36]. As different numerical studies employing phase field for $\mathrm{NiTi}$ and other metallic or alloy systems show [32,34,36,63], in 2-D thin films either two or four inplane B19' variants can evolve (nucleate and grow) simultaneously. Further, the evolution of different variants in 2-D simulation cells depends on its crystallographic orientation, initial condition and temperature, and loading history. As an example, previous studies on 2-D [100], [001] NiTi simulation cells predict evolution of only two in plane B19' variants (variant 1 and 2) $[32,34,63]$. On the other hand, a recent relevant study [36] on [111] orientation of 2-D NiTi simulation cells shows the possibility of evolution of two or four in plane B19' variants and reports some cases observed only in 2-D thin films and not observed in the bulk. Such crystallographic orientation dependent evolution of microstructure in 2-D NiTi thin films can be attributed to the absence of the third direction, which prevents the development of strain components (both axial and shear strain in B2 to B19' transformation) in that third direction. Thereby, in 2-D NiTi simulation cells, based on the crystallographic orientation, the evolution of certain B19' variants are facilitated, while others are suppressed. In this present study, 2-D NiTi thin films with [110], [001] orientation are considered. Due to the presence of the [110] direction, the simulation cell allows the development of the axial and shear components of internal strain during the B2 to B19' phase transformation process and thus the cells are capable in accommodating at least fore B19' variants at the same time.

Figure-9 shows the evolution (formation and growth) of different B19' variants and twines, obtained from phase field (PhF) simulation. Because of the [110], [001] orientations in 2-D simulation cells, the transformation strain tensor for any variant needs to be orientated properly. Thus, before using the transformation strain tensors (adopted from previous studies [31,36,6467]) they are rotated through the rotation matrix $R_{(110)}$ i.e. $\varepsilon_{i}^{0}$ is converted into $R_{(110)} \varepsilon_{i}^{0} R_{(110)}^{T}$ $[31,34,36]$. Some details about this are provided in the supplementary material. Further, at any instant of time, the spatial evolution of any B19' variants and B 2 phase are determined through the quantity $\sum_{i=1}^{12} \Psi_{i}(\mathbf{x}, t) \times i$, which becomes approximately $i$ for the $i^{\text {th }}$ variant of the B19' phase and 0 for the B2 phase. However, consideration of stochasticity in the phase field model causes small deviation $(\approx \pm 0.1)$ in the corresponding values [31,36].

A $1024 \mathrm{~nm} \times 1024 \mathrm{~nm}$ thin film simulation cell is considered. Results are shown for three different temperatures i.e. $\mathrm{T}=320, \mathrm{~T}=350$ and $\mathrm{T}=380 \mathrm{~K}$. Microstructural analysis shows that only four of the different B19' variants (variants 1, 2, 6 and 8 of B19') can be developed periodically in the 2-D simulation cell of thin film [31,36] and all of them can coexist with austenite B2. Since the microstructure evolves periodically within the simulation cell, zoomed-in views are shown in Fig. 9. Observation of only four B19' variants is because of the consideration of 2-D simulation cell resembling a thin film. Absence of the third dimension creates inconsistency in the self-accommodation of all the martensite variants and thus only four in plane variants are observed. For these four B19' phase variants, the presently adopted 
2-D NiTi simulation cells (in [110] direction) can accommodate both axial and shear strain components of the transformation strain tensor. However the general 3-D case calls for 12 martensite variants. 2-D simulation cells provide a framework for studying the martensitic phase transformation process (evolution and nucleation of different phases) in NiTi thin films, which finds many applications [68-73]. Furthermore, previous studies in this field report similar type of microstructure evolution [31,36]. Similar microstructure (with fluctuations) is transferred into the larger spatial domain through the multiscale coupling approach via CWM, not shown here for brevity.
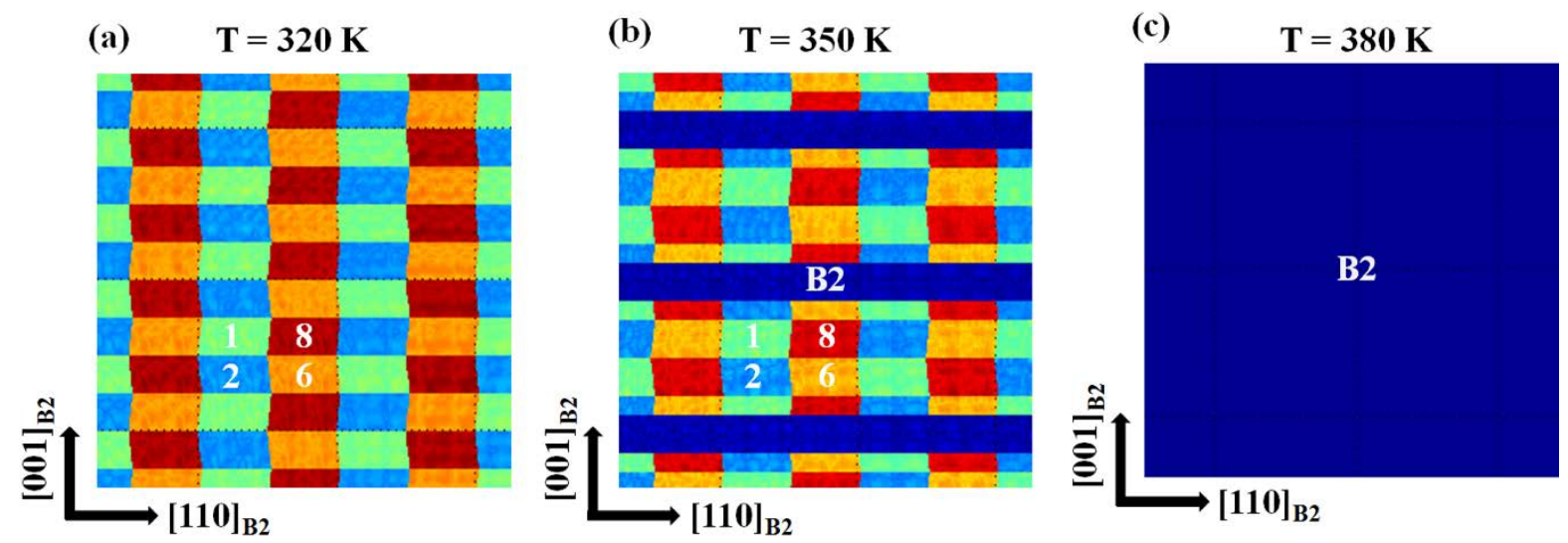

Figure-9: Microstructure of NiTi SMA (zoomed view) due to the thermally induced phase transformation at different temperature (a) $\mathrm{T}=320 \mathrm{~K}$, (b) $\mathrm{T}=350 \mathrm{~K}$ and (c) $\mathrm{T}=380 \mathrm{~K}$. Four different variants of B19' and B2 phase are shown here.

Figure 10 shows the martensite phase fraction as a function of temperature predicted by PhF, from the experiments, and predicted by the CWM method. The phase field simulation size here is $1024 \mathrm{~nm} \times 1024 \mathrm{~nm}$, and, this size is large enough i.e. beyond the size where size effects are predominant. It clearly demonstrates the ability of the CWM method to transfer the trend as well as the uncertainties of phase fraction to large scales i.e. in to the continuum level, through multiple scale coupling method via CWM.
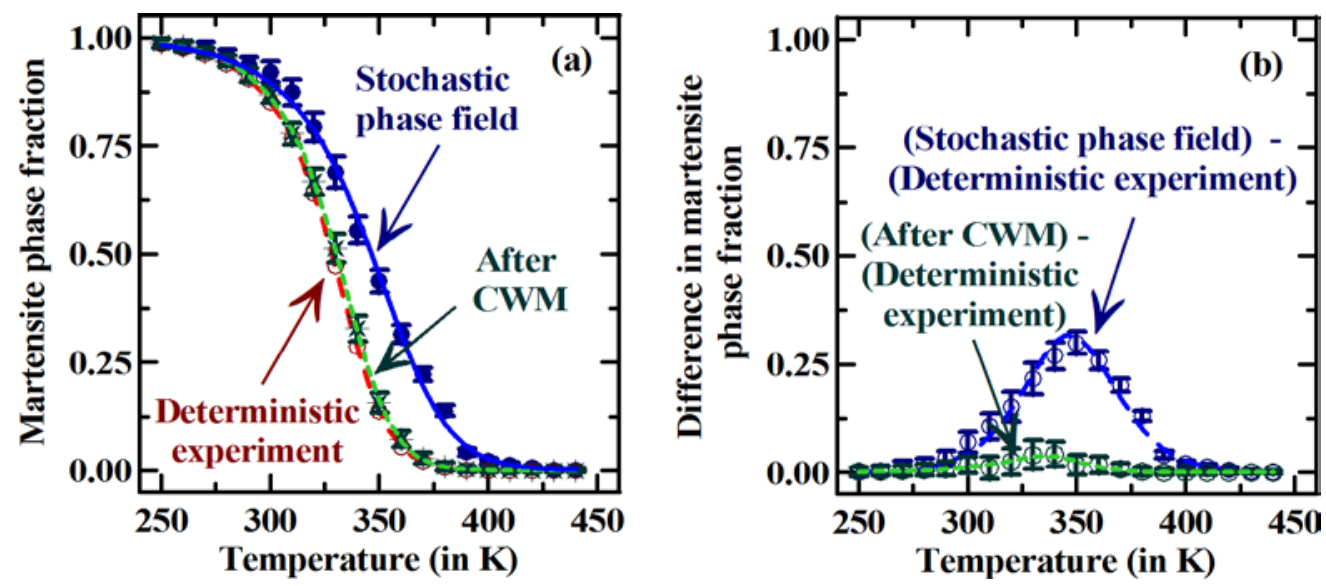

Figure-10: (a) Variation of martensite phase fraction with respect to the temperature from phase field simulation (stochastic, $1024 \mathrm{~nm}$ cell size), deterministic experiment-based, and after CWM. (b) Difference in martensite phase fraction with respect to temperature. 
To demonstrate the accuracy of the CWM method in transferring stochasticity form fine to coarse scales, different statistical moments of martensite phase fraction with respect to temperature are shown in Figure 11. An important point is that the martensite phase fraction is spatially correlated, with a clear correlation distance, which practically denotes an approximate maximum size of NiTi shape memory alloy (SMA) simulation cell or sample above which size effects seize. Interestingly, the correlation distance varies with temperature, and the CWM method captures such a dependency accurately. It is noted that the adopted sample size ( $1024 \mathrm{~nm}$ ) for phase field simulation is larger than the maximum correlation distance at any temperature (approximately $500 \mathrm{~nm}$ ) and therefore designates the beginning of the end of size effects and a minimum size for the coupling process.
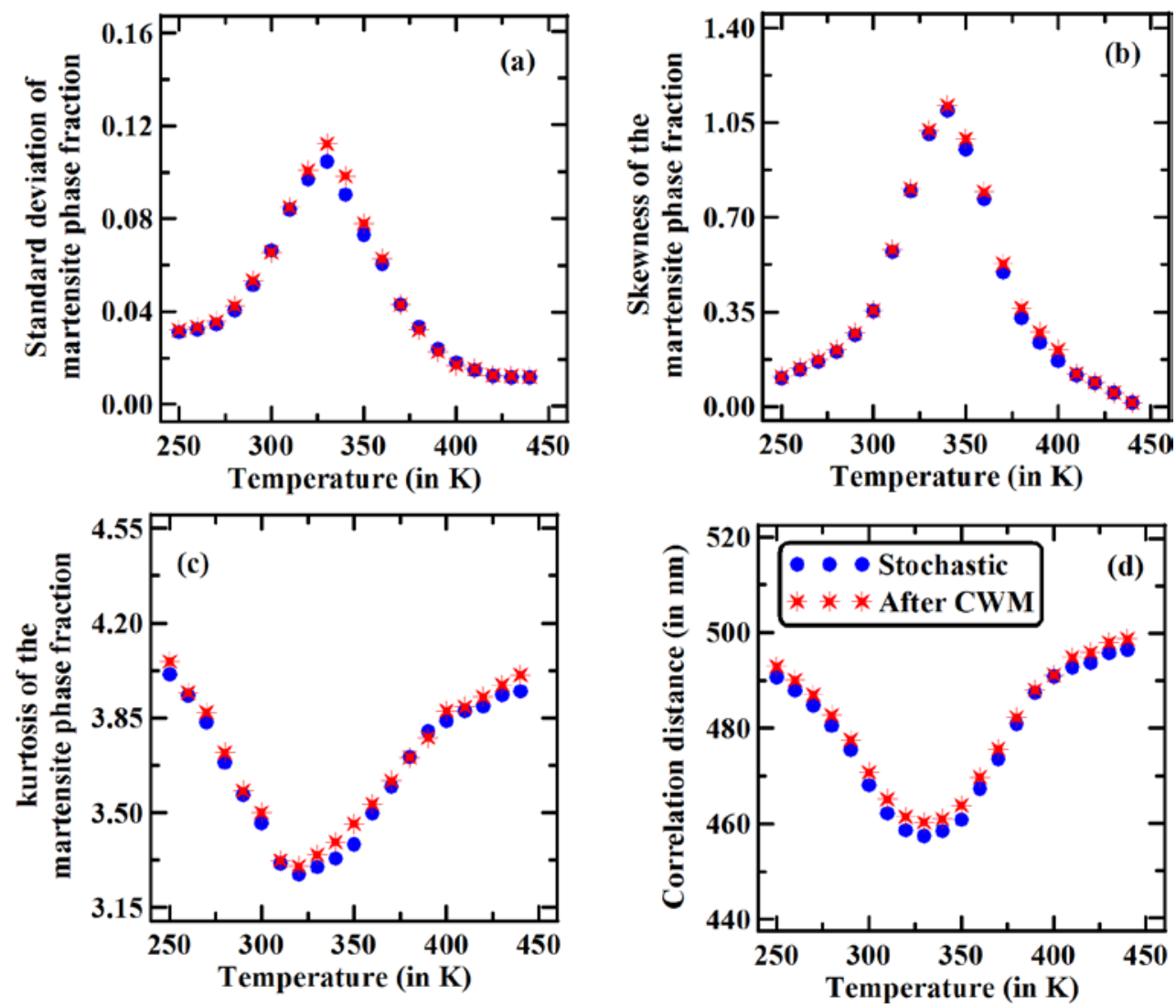

Figure-11: Variation of different statistical measures (a) standard deviation, (b) skewness, (c) kurtosis and (d) correlation distance of martensite phase fraction with respect to temperature.

Finally, the quantified uncertainties on the spatial evolution of thermally induced martensite phase fraction are used to study their effects on stress-strain response and stress induced martensite phase fraction evolution of NiTi. For this, an established strain rate dependent thermomechanical constitutive model for NiTi is adopted, i.e. that of Helm and Haupt [74]. A brief review on this constitutive model is provided in the supplementary material and details appear in [75]. Of course, the martensite phase fraction used in the constitutive model is obtained from the present study, noting that in [74] it is assumed that the thermally induced martensite phase fraction varies linearly with temperature. However, as demonstrated herein, based on experimental studies [43], phase field simulations [31,33,34] and molecular dynamics simulations $[25,27]$ the martensite phase fraction varies exponentially with temperature. The total free energy is expressed as a function of the total martensite phase fraction, thus the dependency of the phase fraction on temperature does not influence the free energy and 
thermodynamics laws [74]. With the thermally induced martensite phase fraction and its associated uncertainties obtained from multiscale coupling, the stress-strain response and stress induced martensite phase fraction of NiTi at different temperatures is shown in Figure 12. Figs. 12a1-c1 show the stress-strain response of NiTi SMA at different temperature such as $\mathrm{T}=320 \mathrm{~K}, \mathrm{~T}=350 \mathrm{~K}$ and $\mathrm{T}=380 \mathrm{~K}$, whereas Figs. $12 \mathrm{a} 2-\mathrm{c} 2$ show the variation of stress induced martensite phase with applied strain at these temperatures. For each temperature, five curves are plotted, i.e. at mean phase fraction value and mean with two different levels of uncertainties ( $\mu, \mu \pm \sigma$ and $\mu \pm 2 \sigma$ ). It can be observed that within the transformation temperature zone (such as $\mathrm{T}=320 \mathrm{~K}$ and $\mathrm{T}=350 \mathrm{~K}$ ) uncertainty in the thermally induced martensite phase fraction has a pronounced effect on the stress-strain response and stress induced martensite phase fraction evolution. However, such effect diminishes near and beyond the upper end of the transformation temperature zone (such as $\mathrm{T}=380 \mathrm{~K}$ ).
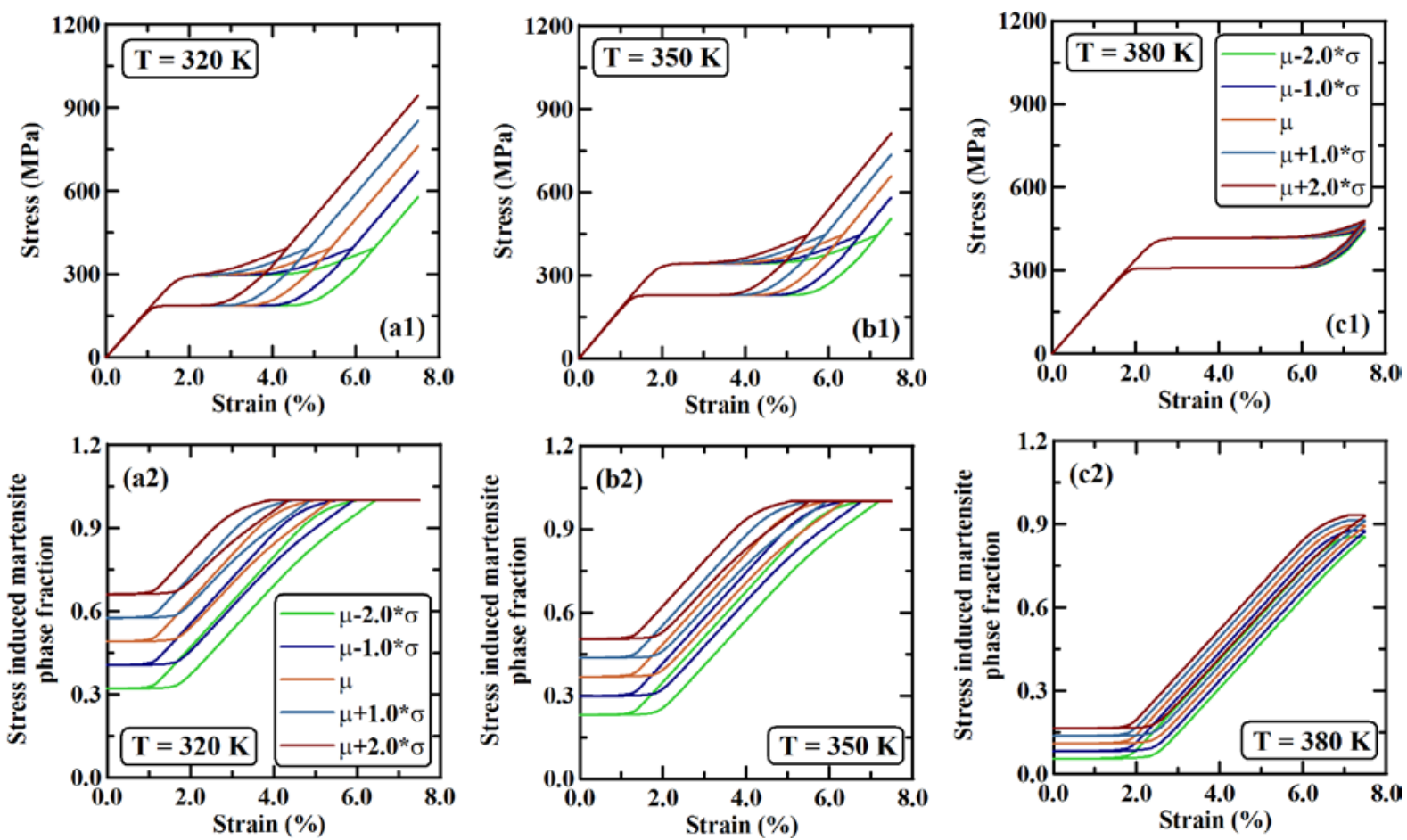

Figure-12: Stress-strain response curve of NiTi SMA at different temperature (a1) $\mathrm{T}=320 \mathrm{~K}$ , (b1) $\mathrm{T}=350 \mathrm{~K}$ and (c1) $\mathrm{T}=380 \mathrm{~K}$. Stress induced martensite phase fraction as a function of applied strain at (a2) $\mathrm{T}=320 \mathrm{~K}$, (b2) $\mathrm{T}=350 \mathrm{~K}$ and (c2) $\mathrm{T}=380 \mathrm{~K}$.

\section{Conclusions}

A multiscale and multiphysics framework for modeling the thermally induced martensite phase fraction evolution in NiTi shape memory alloys is presented. The lattice Boltzmann method (LBM) is employed for simulating the heat transfer (diffusion) process. At meso/ fine scales the time dependent Ginzburg-Landau (TDGL) stochastic phase field simulation method is used for martensite phase fraction evolution, whereas at coarse scales experimental deterministic measurements are used. Multiscale coupling of the data at the range of scales from fine to coarse is achieved through the compound wavelet matrix (CWM) method. The coupling includes a novel process for predicting wavelet coefficients, which preserves the spatial correlation characteristics of the martensite phase fraction. The CWM method shows accurate transfer of statistical information from fine to coarse scales. The multiscale coupling considers 
the size effects in thermally induced martensite phase fraction, which verifies each of the simulation methods used. To robustly address size effects, molecular dynamics simulations are performed in addition to the stochastic phase field simulations and availability of coarse scale experimental data. It is a major conclusion that size effects are important for choosing an appropriate size of NiTi sample for the multiscale coupling. The effects of uncertainties in martensite phase fraction on the macroscopic stress-strain response of NiTi are quantified, and it is concluded that such effects are quantitatively important and have a strong influence on NiTi's constitutive properties. In this sense, it is shown that the spatial correlations in martensite phase fraction is saliently behind the size effects. Finally, based on this and relevant studies it is concluded that the martensite phase fraction varies exponentially with temperature, in contrast to the linear relation typically assumed in macroscopic constitutive relations for SMAs. As far as future work is concerned, the interplay of grain size in polycrystalline SMAs and cell/specimen size is challenging to study yet worth exploring.

\section{Acknowledgement}

The reported exploratory work became possible through the support of SG by the University of Arizona. Support from the THALES/INTERMONU - Project No 68/1117, from the European Union (European Science Foundation - ESF) to GF is acknowledged. Also the authors would like to acknowledge Prof. Ting Zhu (from Woodruff school of mechanical engineering at Georgia Institute of Technology) for providing the interatomic potential parameters.

\section{References}

[1] Mullins M and Dokainish M A 1982 Simulation of the (001) plane crack in $\alpha$-iron employing a new boundary scheme Philos. Mag. A 46 771-87

[2] Tadmor E, Phillips R and Ortiz M 1996 Mixed atomistic and continuum models of deformations in solids J. ACM 7463 4529-34

[3] Kitagawa H, Nakatani A and Shibutani Y 1994 Molecular dynamics study of crack processes associated with dislocation nucleated at the tip Mater. Sci. Eng. A 176 263-9

[4] Frantziskonis G, Mishra S K, Pannala S, Simunovic S, Daw C S, Nukala P, Fox R O and Deymier P A 2006 Wavelet-based Spatiotemporal Multiscaling in Diffusion Problems with Chemically Reactive Boundary Int. J. Multiscale Comput. Eng. 4 755-70

[5] Mishra S K, Muralidharan K, Deymier P A and Frantziskonis G N 2008 Wavelet-Based Spatial Scaling of Coupled Reaction-Diffusion Fields Int. J. Multiscale Comput. Eng. 6 281-97

[6] Muralidharan K, Mishra S K, Frantziskonis G, Deymier P A, Nukala P, Simunovic S and Pannala S 2008 Dynamic compound wavelet matrix method for multiphysics and multiscale problems Phys. Rev. E 77026714

[7] Frantziskonis G, Muralidharan K, Deymier P A, Simunovic S, Nukala P and Pannala S 2009 Time-parallel multiscale/multiphysics framework J. Comput. Phys. 228 8085-92 
[8] Frantziskonis G and Deymier P A 2000 Wavelet methods for analysing and bridging simulations at complementary scales - the compound wavelet matrix and application to microstructure evolution Model. Simul. Mater. Sci. Eng. 8 649-64

[9] Frantziskonis G and Deymier P A 2003 Wavelet-based spatial and temporal multiscaling: Bridging the atomistic and continuum space and time scales Phys. Rev. $B$ 68024105

[10] Vvedensky D D 2004 Multiscale modelling of nanostructures J. Phys. Condens. Matter 16 R1537-76

[11] Sun Q P and He Y J 2008 A multiscale continuum model of the grain-size dependence of the stress hysteresis in shape memory alloy polycrystals Int. J. Solids Struct. 45 386896

[12] Roubíček T 2011 Approximation in multiscale modelling of microstructure evolution in shape-memory alloys Contin. Mech. Thermodyn. 23 491-507

[13] Hariharan G and Kannan K 2014 Review of wavelet methods for the solution of reaction-diffusion problems in science and engineering Appl. Math. Model. 38 799-813

[14] Mishra S K, Muralidharan K, Pannala S, Simunovic S, Daw C S, Nukala P, Fox R O, Deymier P A and Frantziskonis G 2008 Spatiotemporal compound wavelet matrix framework for multiscale / multiphysics reactor simulation: case study of a heterogeneous reaction / diffusion system spatiotemporal compound wavelet matrix framework for multisc Int. J. Chem. React. Engg. 6 1-41

[15] Bi H T and Li J 2004 Multiscale analysis and modeling of multiphase chemical reactors Adv. Powder Technol. 15 607-27

[16] Dollet A 2004 Multiscale modeling of CVD film growth-a review of recent works Surf. Coatings Technol. 177-178 245-51

[17] Bindal A, Khinast J and Ierapetritou M 2003 Adaptive multiscale solution of dynamical systems in chemical processes using wavelets Comput. Chem. Eng. 27 131-42

[18] Stundzia A B and Lumsden C J 1996 Stochastic simulation of coupled reactiondiffusion processes J. Comput. Phys. 127 196-207

[19] Burger R and Ruiz-Baier R 2009 Multiresolution Simulation of Reaction-Diffusion Systems With Strong Degeneracy Bol. Soc. Esp. Mat. Apl. 47 73-80

[20] Vasenkov A V., Fedoseyev A I, Kolobov V I, Choi H S, Hong K-H, Kim K, Kim J, Lee H S and Shin J K 2006 Computational framework for modeling of multi-scale processes Comput. Theor. Nanosci. 3 453-58

[21] Kevrekidis I G, Gear C W, Hyman J M, Kevrekidis P G, Runborg O and Theodoropoulos C 2002 Equation-Free Multiscale Computation: enabling microscopic simulators to perform system-level tasks Commun. Math. Sci. 1 715-62 
[22] Frantziskonis G 2002 Multiscale characterization of materials with distributed pores and inclusions and application to crack formation in an aluminum alloy Prob. Eng. Mech. 17 359-67

[23] Frantziskonis G 2002 Wavelet-based analysis of multiscale phenomena: Application to material porosity and identification of dominant scales Prob. Eng. Mech. 17 349-57

[24] Lai W S and Liu B X 2000 Lattice stability of some Ni-Ti alloy phases versus their chemical composition and disordering J. Phys. Condens. Matter 12 L53-60

[25] Zhong Y, Gall K and Zhu T 2011 Atomistic study of nanotwins in NiTi shape memory alloys J. Appl. Phys. 110033532

[26] Mutter D and Nielaba P 2010 Simulation of structural phase transitions in NiTi Phys. Rev. B 82224201

[27] Mirzaeifar R, Gall K, Zhu T, Yavari A and Desroches R 2014 Structural transformations in NiTi shape memory alloy nanowires J. Appl. Phys. 115

[28] Zhong Y, Gall K and Zhu T 2012 Atomistic characterization of pseudoelasticity and shape memory in NiTi nanopillars Acta Mater. 60 6301-11

[29] Plimpton S 1995 Fast Parallel Algorithms for Short-Range Molecular Dynamics J. Comput. Phys. 117 1-19

[30] Mutter D and Nielaba P 2011 Simulation of the thermally induced austenitic phase transition in NiTi nanoparticles Eur. Phys. J. B 84 109-13

[31] Zhong Y and Zhu T 2014 Phase-field modeling of martensitic microstructure in NiTi shape memory alloys Acta Mater. 75 337-47

[32] Levitas V I, Lee D W and Preston D L 2010 Interface propagation and microstructure evolution in phase field models of stress-induced martensitic phase transformations Int. J. Plast. 26 395-422

[33] Heo T W and Chen L Q 2014 Phase-field modeling of displacive phase transformations in elastically anisotropic and inhomogeneous polycrystals Acta Mater. 76 68-81

[34] Ahluwalia R, Quek S S and Wu D T 2015 Simulation of grain size effects in nanocrystalline shape memory alloys J. Appl. Phys. 117

[35] Wang Y and Khachaturyan A G 1997 Three-dimensional field model and computer modeling of martensitic transformations Acta Mater. 45 759-73

[36] Yang L and Dayal K 2010 Formulation of phase-field energies for microstructure in complex crystal structures Appl. Phys. Lett. 96 081916-9

[37] Wang Y and Li J 2010 Phase field modeling of defects and deformation Acta Mater. 58 1212-35 
[38] Steinbach I and Shchyglo O 2011 Phase-field modelling of microstructure evolution in solids: Perspectives and challenges Curr. Opin. Solids State Mater. Sci. 15 87-92

[39] Salman O U, Finel A, Delville R and Schryvers D 2012 The role of phase compatibility in martensite J. Appl. Phys. 111

[40] Yeddu H K, Lookman T and Saxena A 2013 Strain-induced martensitic transformation in stainless steels: A three-dimensional phase-field study Acta Mater. 61 6972-82

[41] Dhote R P, Fabrizio M, Melnik R N V and Zu J 2013 Hysteresis phenomena in shape memory alloys by non-isothermal Ginzburg-Landau models Commun. Nonlinear Sci. Numer. Simul. 18 2549-61

[42] Gao Y, Zhou N, Wang D and Wang Y 2014 Pattern formation during cubic to orthorhombic martensitic transformations in shape memory alloys Acta Mater. 68 93105

[43] Zotov N, Marzynkevitsch V and Mittemeijer E J 2014 Evaluation of kinetic equations describing the martensite-austenite phase transformation in NiTi shape memory alloys J. Alloys Compd. 616 385-93

[44] Wu K H, Shi J D, Yang F and Pu Z J 1996 Kinetic model of thermoelastic martensite transformation in NiTi and NiMn based shape memory alloys MRS Symp. Proc. 398 537-42

[45] Lai B K, Kahn H, Phillips S M, Akase Z and Heuer A H 2011 Quantitative phase transformation behavior in TiNi shape memory alloy thin films J. Mater. Res. 19 282233

[46] Moelans N, Blanpain B and Wollants P 2008 An introduction to phase-field modeling of microstructure evolution Comput. Coup. Phase Diag. Thermochem. 32 268-94

[47] Mishra S K and De A 2013 Coupling of reaction and hydrodynamics around a reacting block modeled by lattice Boltzmann method (LBM) Comput. Fluids 71 91-7

[48] De A and Mishra S K 2014 Simulation of chemical reactions induced by droplet in a phase separating media using lattice Boltzmann-kinetic Monte-Carlo framework Comput. Fluids 89 133-42

[49] Shan X and Doolen G D 1996 Diffusion in a multicomponent lattice Boltzmann equation model. Phys. Rev. E 54 3614-20

[50] Frantziskonis G N 2011 Lattice Boltzmann method for multimode wave propagation in viscoelastic media and in elastic solids Phys. Rev. E 83066703

[51] Chai Z and Zhao T S 2013 Lattice Boltzmann model for the convection-diffusion equation Phys. Rev. E 87063309 
[52] Mishra S K, Deymier P A, Muralidharan K, Frantziskonis G, Pannala S and Simunovic S 2010 Modeling the coupling of reaction kinetics and hydrodynamics in a collapsing cavity Ultrason. Sonochem. 17 258-65

[53] Peng Y, Shu C and Chew Y T 2003 Simplified thermal lattice Boltzmann model for incompressible thermal flows Phys. Rev. E 68026701

[54] He X, Chen S and Doolen G D 1998 A Novel Thermal Model for the Lattice Boltzmann Method in Incompressible Limit J. Comput. Phys. 146 282-300

[55] Zanotti C, Giuliani P, Bassani P, Zhang Z and Chrysanthou A 2010 Intermetallics 18 $14-21$

[56] Zanotti C, Giuliani P and Chrysanthou A 2012 Martensitic-Austenitic phase transformation of Ni-Ti SMAs: Thermal properties Intermetallics 24 106-14

[57] Zanotti C, Giuliani P, Riva G, Tuissi A and Chrysanthou A 2009 Thermal diffusivity of Ni-Ti SMAs J. Alloys Compd. 473 231-7

[58] D’OrazioA, Succi S and Arrighetti C 2003 Lattice boltzmann simulation of open flows with heat transfer Phys. Fluids 15 2778-81

[59] Wang J, Wang M and Li Z 2007 A lattice Boltzmann algorithm for fluid-solid conjugate heat transfer Int. J. Therm. Sci. 46 228-34

[60] Gur S, Danielson T, Xiong Q, Hin C, Pannala S, Frantziskonis G, Savara A and Daw C S 2016 Wavelet-based surrogate time series for multiscale simulation of heterogeneous catalysis Chem. Eng. Sci. 144 165-75

[61] Mutter D and Nielaba P 2013 Simulation of the shape memory effect in a NiTi nano model system J. Alloys Compd. 577 S83-7

[62] Frantziskonis G N 2013 Multiscale unified prediction of size / scale and Hall-Petch effects in the mechanics of polycrystalline materials J. Mech. Behav. Mater. 22 67-71

[63] Dhote R P, Melnik R N V and Zu J 2014 Dynamic multi-axial behavior of shape memory alloy nanowires with coupled thermo-mechanical phase-field models Meccanica 49 1561-75

[64] Hane K F 1999 Bulk and thin film microstructures in untwinned martensites J. Mech. Phys. Solids 47 1917-39

[65] Hane K F and Shield T W 1999 Microstructure in the cubic to monoclinic transition in Titanium-Nickel shape memory alloys Acta Mater. 47 2603-17

[66] James R D and Hane K F 2000 Martensitic transformations and shape memory materials Acta Mater. 48 197-222

[67] Hane K F and Shield T W 2000 Microstructure in the cubic to trigonal transition Mater. Sci. Eng., A 291 147-59 
[68] Bendahan M, Aguir K, Seguin J L and Carchano H $1999 \mathrm{NiTi}$ thin films as a gate of M.O.S. capacity sensors Sens. Actuators, A: Physical 74 242-5

[69] Kohl M, Dittmann D, Quandt E and Winzek B 2000 Thin film shape memory microvalves with adjustable operation temperature Sens. Actuators, A: Physical 83 2149

[70] Fu Y Q, Zhang S, Wu M J, Huang W M, Du H J, Luo J K, Flewitt A J and Milne W I 2006 On the lower thickness boundary of sputtered TiNi films for shape memory application Thin Solid Films 515 80-6

[71] Chan P M, Chung C Y and Ng K C 2008 NiTi shape memory alloy thin film sensor micro-array for detection of infrared radiation J. Alloys Compd. 449 148-51

[72] Choudhary N, Kharat D K and Kaur D 2011 Structural, electrical and mechanical properties of magnetron sputtered NiTi/PZT/TiOx thin film heterostructures Surf. Coat. Technol. 205 3387-96

[73] Lai B K, Kahn H, Phillips S M, Akase Z and Heuer A H 2004 Quantitative phase transformation behavior in TiNi shape memory alloy thin films J. Mater. Res 192822 33

[74] Helm D and Haupt P 2003 Shape memory behaviour: modelling within continuum mechanics Int. J. Solids Struct. 40 827-49

[75] Gur S, Mishra S K and Frantziskonis G N 2015 Thermo-mechanical strain ratedependent behavior of shape memory alloys as vibration dampers and comparison to conventional dampers J. Intell. Mater. Syst. Struct. 27 1250-64 